\title{
Glutathione Peroxidase-1 Suppresses the Unfolded Protein Response upon Cigarette Smoke Exposure
}

\author{
Patrick Geraghty, ${ }^{1,2}$ Nathalie Baumlin, ${ }^{3}$ Matthias A. Salathe, ${ }^{3}$ \\ Robert F. Foronjy, ${ }^{1,2}$ and Jeanine M. D'Armiento ${ }^{4}$ \\ ${ }^{1}$ Division of Pulmonary \& Critical Care Medicine, Department of Medicine, \\ State University of New York Downstate Medical Center, Brooklyn, NY, USA \\ ${ }^{2}$ Department of Cell Biology, State University of New York Downstate Medical Center, Brooklyn, NY, USA \\ ${ }^{3}$ Division of Pulmonary, Allergy, Critical Care, and Sleep Medicine, University of Miami, Miami, FL, USA \\ ${ }^{4}$ Center for Pulmonary Disease, Department of Anesthesiology, College of Physicians and Surgeons, \\ Columbia University, New York, NY, USA \\ Correspondence should be addressed to Patrick Geraghty; patrick.geraghty@downstate.edu
}

Received 18 August 2016; Revised 19 October 2016; Accepted 31 October 2016

Academic Editor: Karen Ridge

Copyright (C) 2016 Patrick Geraghty et al. This is an open access article distributed under the Creative Commons Attribution License, which permits unrestricted use, distribution, and reproduction in any medium, provided the original work is properly cited.

\begin{abstract}
Oxidative stress provokes endoplasmic reticulum (ER) stress-induced unfolded protein response (UPR) in the lungs of chronic obstructive pulmonary (COPD) subjects. The antioxidant, glutathione peroxidase-1 (GPx-1), counters oxidative stress induced by cigarette smoke exposure. Here, we investigate whether GPx-1 expression deters the UPR following exposure to cigarette smoke. Expression of ER stress markers was investigated in fully differentiated normal human bronchial epithelial (NHBE) cells isolated from nonsmoking, smoking, and COPD donors and redifferentiated at the air liquid interface. NHBE cells from COPD donors expressed heightened ATF4, XBP1, GRP78, GRP94, EDEM1, and CHOP compared to cells from nonsmoking donors. These changes coincided with reduced GPX-1 expression. Reintroduction of GPX-1 into NHBE cells isolated from COPD donors reduced the UPR. To determine whether the loss of GPx-1 expression has a direct impact on these ER stress markers during smoke exposure, Gpx$1^{-/-}$mice were exposed to cigarette smoke for 1 year. Loss of Gpx-1 expression enhanced cigarette smoke-induced ER stress and apoptosis. Equally, induction of ER stress with tunicamycin enhanced antioxidant expression in mouse precision-cut lung slices. Smoke inhalation also exacerbated the UPR response during respiratory syncytial virus infection. Therefore, ER stress may be an antioxidant-related pathophysiological event in COPD.
\end{abstract}

\section{Introduction}

Chronic obstructive pulmonary disease (COPD) is the third leading cause of death in the US [1], with cigarette smoking being the most important environmental risk factor. Cigarette smoke inhalation alters the expression profile of oxidants and antioxidants in the lungs and produces an enormous oxidant burden [2]. Antioxidant enzymes counter this oxidative stress [2] and deter lung inflammation responses by targeting multiple signaling pathways [3]. Detoxifying reactive oxygen species (ROS) is a therapeutic strategy to limit tissue damage in cigarette smoke-induced diseases [3]. Recently, cigarette smoke-mediated oxidative stress was shown to induce endoplasmic reticulum (ER) stress [4]. However, the ability of antioxidants to counter ER stress has not been fully characterized.

It is well established that cigarette smoke induces ER stress which activates the unfolded protein response (UPR) [5-9]. However, COPD is a complex heterogeneous disease and the significance and intensity of the UPR during the disease is unknown. The UPR is a complex stress response program that modulates multiple cellular responses and survival, via regulation of protein synthesis, folding, and degradation [10]. Three major pathways of the UPR have been characterized: (i) PKR-like ER kinase (PERK)/eIF2 $\alpha /$ activating transcription factor (ATF) 4/CHOP, (ii) inositol-requiring 
enzyme 1 (IRE1)/X-box binding protein 1 (XBP1), and the (iii) ATF6 pathway [11]. These pathways regulate ER chaperone responses and reduce protein translation following cellular stress [12]. However, persistent ER stress results in significant expression of the proapoptotic gene C/EBP homologous protein (CHOP), resulting in cell death [11]. We previously demonstrated that smoke exposure triggers a minor ER stress response in primary cells and rodent animal models [5]. However, a secondary insult may be required to provoke a significant smoke-induced UPR.

Our group demonstrated that overexpression of glutathione peroxidase- (GPx-) 1, a member of the selenoprotein family, prevents cigarette smoke-induced air space enlargement in mice [13]. GPx-1 is the most abundant GPx isoform in eukaryotic cells and deficiency of this enzyme can lead to endothelial dysfunction [14] and apoptosis [15]. GPX-1 deficiency has also been implemented as a contributor to atherosclerosis [16]. Gpx-1 deficient mice exposed to cigarette smoke are more susceptible to cigarette smoke-induced lung inflammation and emphysema [13, 17]. Oxidative stress induces ER stress [4] and increased expression of ER stress markers is observed in the lungs of smokers [8]. GPx-1 expression, however, is reduced in COPD lungs [13]. Thus, we speculate that GPx-1 could modulate ER stress responses linked to the pathogenesis of COPD.

In view of the potential association between $\mathrm{GPx}-1$ and cigarette smoke-mediated UPR, we explored whether the loss of GPx-1 expression enhanced the UPR that contributes to lung cell injury and death. Using normal human bronchial epithelial (NHBE) cells from nonsmokers, smokers, and COPD subjects, we found that ER stress markers were significantly elevated in cells isolated from COPD subjects and this increase coincided with reduced GPx-1 expression. Reintroducing GPx-1 into these cells blunted the UPR. To determine if GPx-1 depletion in the lung directly enhances ER stress, $G p x-1^{-1-}$ mice were exposed to cigarette smoke for 1 year. Interestingly, the loss of GPx-1 expression activated all three branches of the UPR, PERK/eIF $2 \alpha /$ ATF4/CHOP, IRE1/XBP1, and ATF6. This UPR coincided with elevated lung cell death in $G p x-1^{-/-}$mice following smoke exposure. Interestingly, precision-cut lung slices (PCLS) from mice had elevated GPx proteins following induction of ER stress. These findings indicate that the altered GPx-1 expression in COPD lungs contributes to heightened ER stress. In addition, early induction of ER stress induces an antioxidant response to counter oxidative stress, thereby limiting the UPR.

\section{Materials and Methods}

2.1. Human Primary Airway Cells. NHBE cells from nonsmokers, smokers, and COPD patients were isolated from human lungs. Lungs were obtained from organ donors whose lungs were rejected for transplant (see Table 1 for demographics). Consent for research was obtained by the Life Alliance Organ Recovery Agency of the University of Miami. All consents were IRB-approved and conformed to the Declaration of Helsinki. For lungs from donors with COPD, the diagnosis was listed in the chart before the death of the donor and we
TABLE 1: Donor demographics for epithelial cell.

\begin{tabular}{lccc}
\hline & Nonsmokers & Smokers & COPD donors \\
\hline Number & 9 & 6 & 9 \\
Age (years) & $36.3 \pm 14.8$ & $36.0 \pm 13.2$ & $49 \pm 6.8$ \\
Gender (male/female) & $2 / 7$ & $3 / 3$ & $5 / 4$ \\
$\begin{array}{l}\text { Race } \\
\text { (Caucasian/African }\end{array}$ & $77.8 \% / 22.2 \%$ & $100 \% / 0 \%$ & $88.90 \% / 11.1 \%$ \\
American) & & & \\
Pack years & $0 \pm 0$ & N/A & $48.2 \pm 14.5$ \\
\hline
\end{tabular}

Values are means $\pm \mathrm{SD} . \mathrm{N} / \mathrm{A}=$ not available.

confirmed the macropathological presence of emphysema in these lungs. All COPD subjects had a significant smoking history. NHBE cells isolated from nonsmokers, smokers, and COPD subjects were dedifferentiated through expansion and redifferentiated at an air liquid interface (ALI) on $24 \mathrm{~mm}$ T-clear filters (Costar Corning, Corning, NY, USA) at $37^{\circ} \mathrm{C}, 5 \% \mathrm{CO}_{2}$, as previously described [18]. Cells were collected for protein and RNA analysis. CD45 and CD11C expressions were analyzed which determined a low level of inflammatory-cell contamination and confirmed NHBE cell purity. Fully differentiated NHBE cells from nonsmokers were also exposed to cigarette smoke using a Vitrocell VC-10 smoking robot (Vitrocell Systems GMBH, Waldkirch, Germany). Four cigarettes were smoked according to ISO standard 3308: six puffs per cigarette with a $35 \mathrm{~mL}$ volume per puff and a waiting time between each puff of 60 seconds. NHBE cells were exposed every second day, on three separate days, to 4 cigarettes. RNA was extracted from the NHBE cells for quantitative PCR (qPCR) analysis. A subset of NHBE cells from COPD subjects was protein transfected $2 \mu \mathrm{g}$ human GPX-1 protein or human albumin (both from Signal Aldrich) using Pierce transfection reagent (ThermoFisher Scientific) as previously described $[13,19]$. RNA and protein were collect 24 hours later. Human RSV strain A2 (ATCC, Manassas, VA; \#VR-1540) was infected in NHBE cells as previously described [20]. RNA and protein were collect 24 hours later.

2.2. Animal Models. Gpx-1 $1^{-/-}$mice were bred in $\mathrm{C} 57 \mathrm{BL} / 6 \times$ CBA/J background. Eight-week-old wild type and $G p x-1^{-/-}$ mice were used for all experiments. All mice were maintained in a specific pathogen-free facility at Columbia University. Both male and female mice, 8-week-old, were used at the initiation point for all experiments and each experimental parameter had at least 10 animals per group. Mice were exposed to cigarette smoke in a chamber (Teague Enterprises, Davis, CA, USA) for four hours a day, five days per week at a total particulate matter concentration of $80 \mathrm{mg} / \mathrm{m}^{3}$. Smoke exposure was continued for 1 year. The University of Kentucky reference research cigarettes 3R4F (Lexington, KY, USA) were used to generate cigarette smoke. Another group of wild type animals was infected with $1 \times 10^{6}$ pfu of RSV following 6month exposure to room air or cigarette smoke. The institute approved all experiments for Animal Care and Use Committee of Columbia University. This study was performed in strict accordance with the recommendations in the Guide for the 
TABLE 2: TaqMan probe details for gene expression analysis.

\begin{tabular}{|c|c|c|c|c|}
\hline Species & Gene target & NCBI reference sequence & TaqMan assay ID & Product size \\
\hline Human & DDIT3 (CHOP) & NM_001195053.1 & Hs00358796_g1 & 93 \\
\hline Human & ATF4 & NM_001675.2 & Hs00909569_g1 & 68 \\
\hline Human & $X B P 1$ & NM_001079539.1 & Hs00231936_ml & 60 \\
\hline Human & HSPA5 (GRP78) & NM_005347.4 & Hs00607129_gH & 146 \\
\hline Human & HSP90B1 (GRP94) & NM_003299.2 & Hs00427665_g1 & 135 \\
\hline Human & EDEM1 & NM_014674.2 & Hs00976004_m1 & 89 \\
\hline Human & $G P X 1(G P x-1)$ & NM_201397.1 & Hs01028922_g1 & 70 \\
\hline Human & $I L-6$ & NM_000600.3 & Hs00985639_m1 & 66 \\
\hline Human & $A C T B$ & NM_001101.3 & Hs01060665_g1 & 63 \\
\hline Human & PPFIA2 (CD45) & NM_001220473.1 & Hs00170308_m1 & 66 \\
\hline Human & ITGAX (CD11C) & NM_000887.3 & Hs00174217_m1 & 119 \\
\hline Mouse & Ddit3 (Chop) & NM_001290183.1 & Mm01135937_g1 & 92 \\
\hline Mouse & Atf4 & NM_001287180.1 & Mm00515325_g1 & 78 \\
\hline Mouse & $X b p 1$ & NM_001271730.1 & Mm00457357_m1 & 56 \\
\hline Mouse & Hspa5 (Grp78) & NM_001163434.1 & Mm00517691_m1 & 75 \\
\hline Mouse & Hsp90b1 (Grp94) & NM_011631.1 & Mm00441926_m1 & 67 \\
\hline Mouse & Edem 1 & NM_138677.2 & Mm00551797_m1 & 63 \\
\hline Mouse & $G p x-1$ & NM_008160.6 & Mm00656767_g1 & 134 \\
\hline Mouse & $G p x-2$ & NM_030677.2 & Mm00850074_g1 & 147 \\
\hline Mouse & $G p x-3$ & NM_008161.3 & Mm00492427_ml & 99 \\
\hline Mouse & $G p x-4$ & NM_008162.3 & Mm00515041_m1 & 103 \\
\hline Mouse & Sodl & NM_011434.1 & Mm01344233_g1 & 71 \\
\hline Mouse & Actb & AK075973.1 & Mm02619580_g1 & 143 \\
\hline
\end{tabular}

Care and Use of Laboratory Animals of the National Institutes of Health and Institutional Animal Care and Use Committee (IACUC) guidelines.

2.3. Precision-Cut Lung Slices (PCLS). Mouse precision-cut lung slices (PCLS) were prepared as previously described $[21,22]$. Briefly, mice were euthanized, the trachea was cannulated, and the animal was exsanguinated by cutting the jugular vein. The lungs were filled through the cannula with $1.5 \mathrm{~mL}$ low melting-point agarose solution (1.5\% final concentration of agarose in PBS). Lungs were placed on ice for 15 minutes to solidify the agarose. Lobes were separated and tissue cores were prepared of the individual lobes, after which the lobes were sliced at a thickness of $300 \mu \mathrm{m}$ using a Krumdieck tissue slicer (Alabama Research and Development, Munford, AL, USA) in Earle's balanced salt solution (Sigma Aldrich). Tissue slices were incubated in Dulbecco's modified eagle's medium/nutrient mixture F12 HAM solution (Sigma Aldrich) at $37^{\circ} \mathrm{C}$ in a humid atmosphere under $5 \% \mathrm{CO}_{2} / 95 \%$ air. To remove agarose and cell debris, slices were washed every 30 minutes for 2 hours. PCLS were incubated in DMEM supplemented with penicillin $(100 \mathrm{U} / \mathrm{mL})$ and streptomycin $(100 \mu \mathrm{g} / \mathrm{mL})\left(\mathrm{Gibco}^{\circledR}\right.$ by Life Technologies). Slices were cultured at $37^{\circ} \mathrm{C}$ in a humidified atmosphere under $5 \% \mathrm{CO}_{2} / 95 \%$ air in 6 -well tissue culture plates, using 3 slices per well. Slices were treated with $1 \mu \mathrm{M}$ tunicamycin (Sigma Aldrich) for 24 hours. To assess the viability of the PCLS subjected to tunicamycin, lactate dehydrogenase (LDH) released from the PCLS into the incubation medium was analyzed. Maximal LDH release was determined by lysing 3 slices with 1\% Triton X-100 for 30 minutes at $37^{\circ} \mathrm{C}$. $\mathrm{LDH}$ release was determined using an assay form Sigma Aldrich.

2.4. qPCR Analysis. Total RNA was isolated from cells or mouse lung tissue using the Qiagen RNeasy Mini Kit as described by manufactures. Gene transcript levels of mouse and human specific CHOP, ATF4, XBP1, GRP78, GRP94, EDEM1, GPx-1, GPx-2, GPx-3, GPx-4, IL-6, and ACTB were quantified by real-time PCR with the use of an Bio-Rad CFX384 real-time system (Bio-Rad). TaqMan ${ }^{\circledR}$ Gene Expression Assays were purchased from Applied Biosystems (see Table 2 for details). Data is represented as relative quantification (RQ) corrected to ACTB. XBP1 splicing was also examined in NHBE cells using the following primers: $5^{\prime}$-TTA CGA GAG AAA ACT CAT GGC- $3^{\prime}$ and $5^{\prime}$-GGG TCC AAG TTG TCC AGA ATG C-3'. XBP1 PCR products were resolved and run on a $2.5 \%$ agarose gel [23]. 289 and 286 base pair amplicons were generated from unspliced and spliced XBP1, respectively. Percent of XBP1 slicing was examined by densitometry analysis of the unspliced (XBP1u) and spliced (XBP1s) amplicons of XBP1, using Bio-Rad Laboratories Image Lab software (version 4.0, build 16).

2.5. Immunoblotting. Cell monolayers were removed by scrapping in cold phosphate-buffered saline and resuspended 
in $100 \mu \mathrm{L}$ of protein lysis buffer (50 mM HEPES, $\mathrm{pH} 7.5$, $150 \mathrm{mM} \mathrm{NaCl}, 1 \%$ Triton X-100, 1\% glycerol, 1 mM EDTA, $10 \mathrm{mM} \mathrm{NaF}, 2 \mu \mathrm{g} / \mathrm{mL}$ leupeptin, $1 \mu \mathrm{g} / \mathrm{mL}$ pepstatin $\mathrm{A}, 10 \mathrm{mM}$ $\mathrm{Na}_{3} \mathrm{VO}_{4}$, and $1 \mathrm{mM}$ phenylmethylsulfonyl fluoride), and $20 \mu \mathrm{g}$ of protein was separated on $12 \%$ SDS-polyacrylamide gels and transferred to nitrocellulose membranes. Rabbit antibodies against CHOP (Cell Signaling; \#5554), ER degradation-enhancing $\alpha$-mannosidase-like (EDEM) (Santa Cruz Biotechnology; sc-27389), pho-eIF2 $\alpha$ (Ser51) (Cell Signaling; \#9721), eIF2 $\alpha$ (Cell Signaling; \#9722), pho-PERK (Thr980) (Cell Signaling; \#3179), PERK (Cell Signaling; \#5683), XBP-1 (Cell Signaling; \#12782), IRE1 $\alpha$ (Cell Signaling; \#3294), BiP/GRP78 (Cell Signaling; \#3183), GRP94 (Cell Signaling; \#2104), ATF4 (Cell Signaling; \#11815), ATF6 (Abcam; \#ab11909), GPx-1 (Cell Signaling; \#3206), GPx-2 (Abcam; \#ab140130), GPx-3 (Abcam; \#ab27325), GPx-4 (Cell Signaling; \#2104), and $\beta$-actin (Cell Signaling; \#4970) were detected with enhanced chemiluminescence reagents (Pierce). Chemiluminescence detection was performed using the Bio-Rad Laboratories Molecular Imager ChemiDoc XRS+ imaging system. Densitometry was performed on each target and represented as a ratio of pixel intensity compared to total protein or $\beta$-actin, using Bio-Rad Laboratories Image Lab software (version 4.0, build 16).

2.6. Statistical Analysis. Data are expressed as dot plots with the means \pm SEM highlighted. Differences between two groups were compared by Student's $t$ test (two-tailed). Experiments with more than 2 groups were analyzed by 2way ANOVA with Tukey's post hoc test analysis. $p$ values for significance were set at 0.05 and all significant changes were noted with *. All analysis was performed using GraphPad Prism Software (Version 6.0h for Mac OS X).

\section{Results}

3.1. NHBE Cells Isolated from COPD Donors Express More ER Stress Markers than Cells from Smokers. Our group previously demonstrated that undifferentiated NHBE cells have an increased trend in ER stress upon exposure to cigarette smoke extract (CSE) [5]. To examine whether fully differentiated NHBE cells cultured at the air liquid interface (ALI) have an UPR to smoke, NHBE cells from nonsmokers were exposed to 0 (room air, RA) or repeat exposure to four cigarettes (CS) using a Vitrocell VC-10 smoking robot (Figures 1(a)-1(b)). Repeat exposures were performed to maximize smoke stimuli without inducing apoptosis, determined by $\mathrm{LDH}$ release assays (Figure 1(a)). Gene expression of IL-6 was utilized as a positive control for sufficient exposure to smoke [20] (Figure 1(a)). CD45 and CD11C expressions were analyzed but detection was below significant amplification levels thereby confirming low levels of inflammatory-cell contamination (data not shown). Gene expression levels of ATF4, XBP1, GRP78, GRP94, EDEM1, and CHOP were examined. These targets are readouts for the three major pathways of the UPR. No ER stress marker was significantly altered following smoke exposure (Figure 1(b)), as we previously described in submerged cultured NHBE cells [5]. However, when comparing the same ER stress markers in NHBE cells isolated from nonsmokers, smokers, and COPD donors, expressions of ATF4, XBP1, GRP78, GRP94, EDEM1, and CHOP were all increased in cells isolated from COPD subjects (Figure 1(c)). EDEM1 gene expression was significantly enhanced in cells isolated from smokers (Figure 1(c)). There were increased trend changes for ER stress markers in cells from smokers. Protein analysis also confirmed increased expression of ATF4, IRE1 $\alpha$, GRP78, GRP94, EDEM, and CHOP in cells isolated from COPD subjects (Figure 2(a)). Equally, elevated phosphorylation of eIF2 and PERK was observed only in cells isolated from COPD donors (Figure 2(a)).

XBP1 coordinates the adaptive UPR by playing a vital role in maintaining the ER function. Gene expression results showed that NHBE cells isolated from COPD subjects had enhanced XBP1 mRNA splicing compared to cells from nonsmokers and smokers (Figure 2(b)), demonstrating active XBP1 signaling; Protein analysis also confirmed increased expression of XBP1 in cells isolated from COPD donors (Figure 2(b)). Overall, the disease-state predisposes NHBE cells to enhanced ER stress. On the other hand, acute smoke exposure has only a minor impact on ER stress.

3.2. GPx-1 Regulates CHOP Expression in NHBE Cells Isolated from COPD Donors. Since oxidative stress induces ER stress [4], GPx-1 expression is reduced in the COPD lungs [13], and GPx-1 deficiency increases susceptibility to cigarette smokeinduced emphysema $[13,17]$, we examined whether GPx1 expression was altered in fully differentiated NHBE cells isolated from nonsmokers, smokers, and COPD donors (Figure 3). GPX-1 expression was unchanged in NHBE cells from nonsmokers when exposed to cigarette smoke (Figure 3(a)). NHBE cells from nonsmokers and smokers expressed comparable levels of GPx-1 (Figures 3(b)-3(c)). However, cells isolated from COPD subjects had significantly reduced GPx-1 expression, confirmed by q-PCR (Figure 3(b)) and immunoblots (Figure 3(c)). Therefore, the disease-state predisposes NHBE cells to subdued GPx-1 expression.

To determine whether restoring GPx-1 levels in NHBE cells from COPD subjects would reverse heightened UPR, we protein-transfected GPX-1 protein into NHBE cells from COPD subjects. Albumin was transfected as a negative control. Transfection of GPx-1 significantly reduced CHOP gene (Figure 3(d)) and protein (Figure 3(e)) expression in NHBE cells from COPD subjects. Therefore, GPX-1 is a potent regulator of the UPR in the lungs.

3.3. Viral Exacerbations of the Lung Enhance the UPR. To determine whether a second environmental exposure could alter the UPR in our models, we infected NHBE cells and mice with respiratory syncytial virus (RSV). Viral infections have been implicated in the pathogenesis of COPD exacerbations [24, 25] and also trigger the UPR [26]. RSV infected reduced GPx-1 expression and significantly enhanced CHOP expression in NHBE cells from all subject groups (Figures 4(a)-4(b)). Similar changes to CHOP and GPx-1 expression were observed in the lungs of mice infected with RSV (Figures $4(c)-4(d))$. Importantly, prior exposure to cigarette smoke 


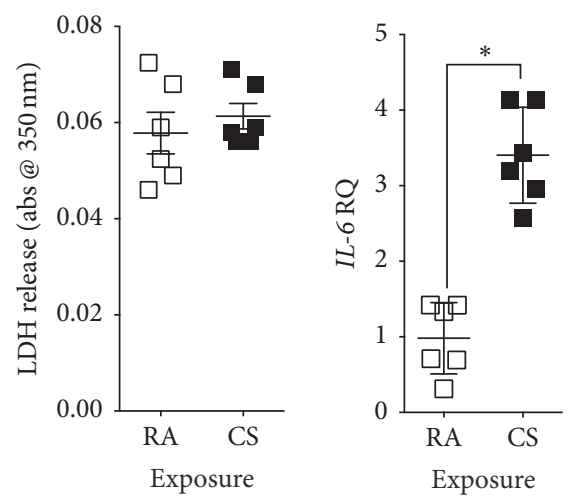

(a)

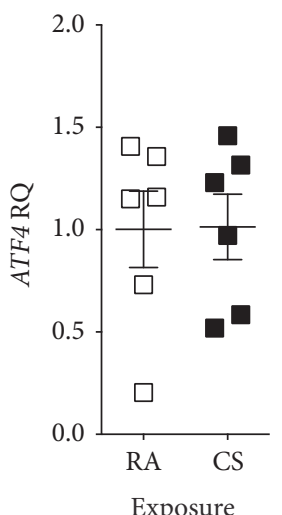

Exposure

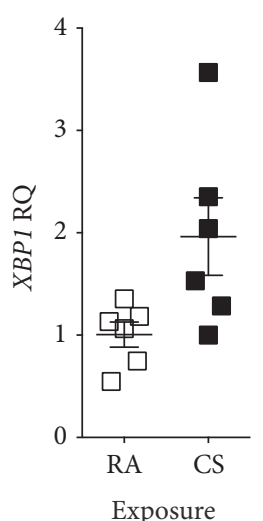

Exposure

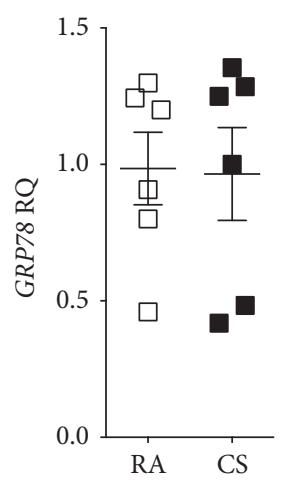

Exposure

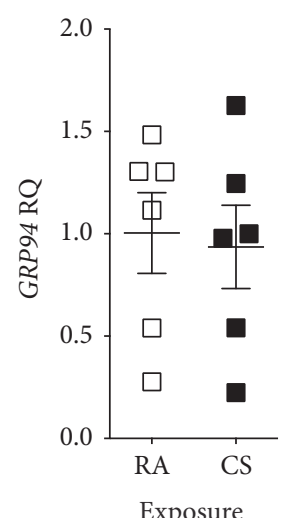

Exposure

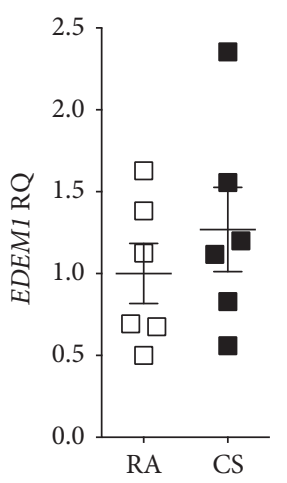

Exposure

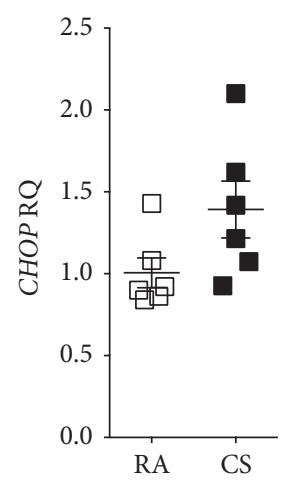

Exposure

(b)

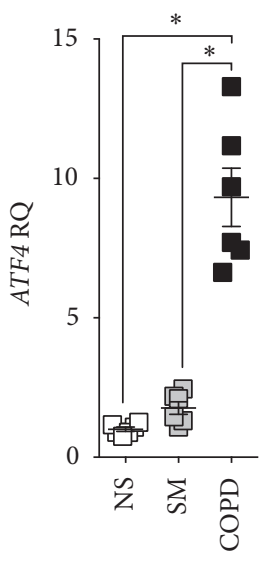

Cell source

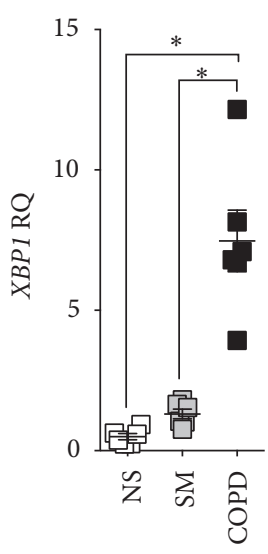

Cell source

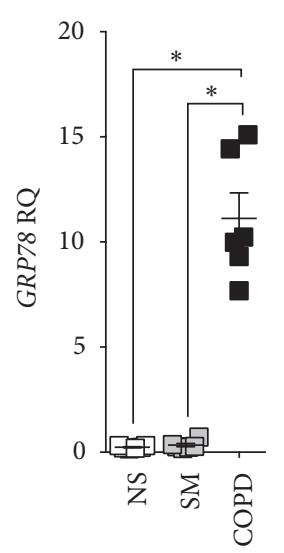

Cell source

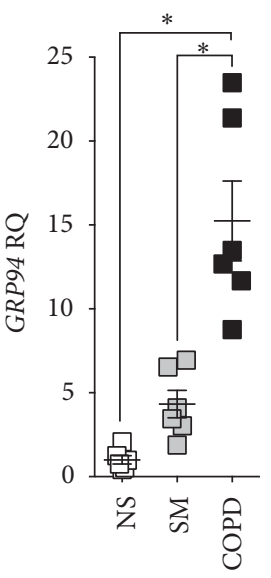

Cell source

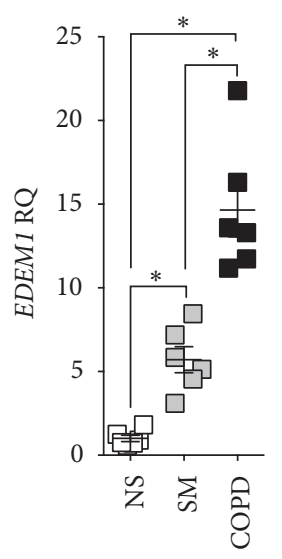

Cell source

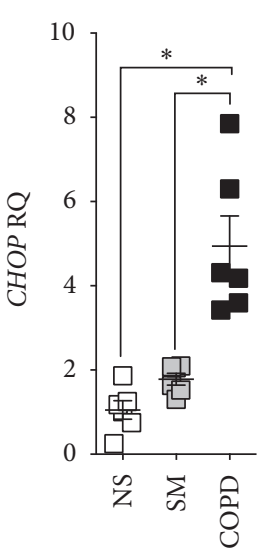

Cell source

(c)

FiguRE 1: NHBE cells isolated from COPD donors have enhanced ER stress responses compared to nonsmokers and smokers. (a) Fully differentiated NHBE cells from nonsmoking individuals $(n=6)$ exposed to room air (RA) or cigarette smoke (CS) from 4 cigarettes every second day (3 exposures) using a Vitrocell VC-10 smoking robot. LDH release into media and IL-6 gene expression were examined. (b) Gene expression of ATF4, XBP1, GRP78, GRP94, EDEM1, and CHOP was examined. (c) Fully differentiated NHBE cells from nonsmoker (NS), smoker (SM), and COPD (COPD) individuals ( $n=6$ donors per group) were examined for gene expression of $A T F 4, X B P 1, G R P 78$, GRP94, EDEM1, and CHOP. Dot plots are represented as relative quantification (RQ) compared to ACTB expression and shown as the mean \pm SEM, where each measurement was performed on 3 independent days on 6 donors/group. $*$ denotes $p$ value $<0.05$, when comparing both treatments connected by a line, determined by Student's $t$-test ( 2 groups) or 2-way ANOVA with Tukey's post hoc test ( $>2$ groups). 

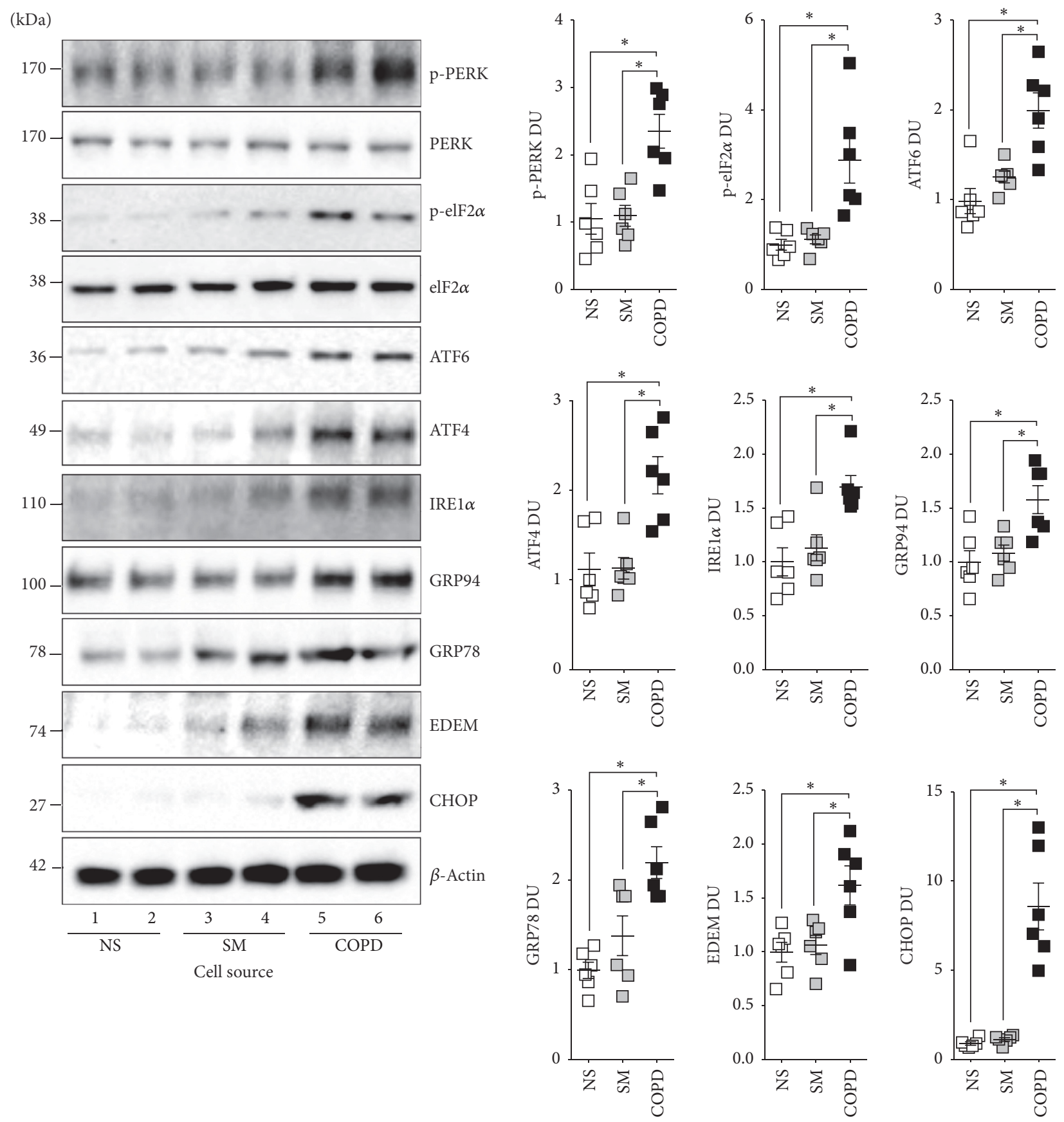

(a)

(bp)

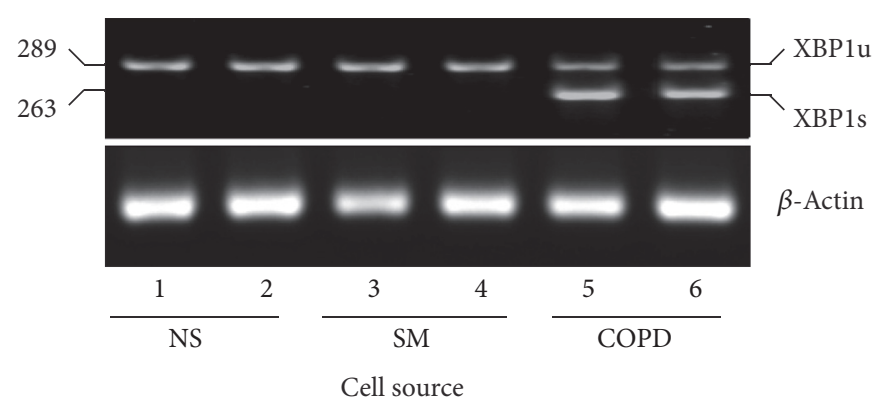

(kDa)

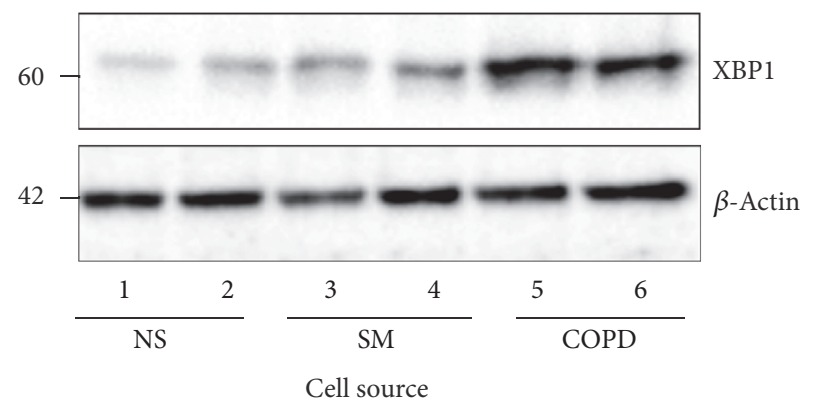

Figure 2: Continued. 


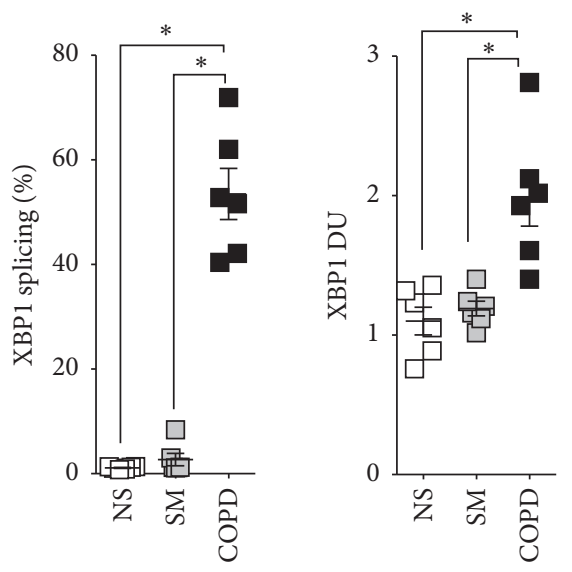

(b)

FIGURE 2: NHBE cells isolated from COPD donors have enhanced ER stress protein responses compared to nonsmokers and smokers. Protein was collected from fully differentiated NHBE cells from nonsmokers (NS), smokers (SM), and COPD (COPD) individuals ( $n=6$ donors per group). (a) Protein expression of ATF6, ATF4, GRP94, GRP78, EDEM, CHOP, and $\beta$-actin was examined by immunoblots. Phosphorylation of elF2 and PERK was also determined. (b) XBP1 splicing was examined on XBP1 amplified cDNA from NHBE cells from nonsmoker (NS), smokers (S), and COPD (COPD) individuals. Protein expression of XBP1 and $\beta$-actin was examined by immunoblots. (a)-(b) For each blot or gel, every lane represents an individual cell donor. Densitometry analysis was performed of XBP1s from DNA gels and other targets by analyzing immunoblots. XBP1 slicing was scored as percent of XBP1s of total XBP1. Dot plots are represented as densitometry units (DU) of pixel intensity expressed as a ratio to $\beta$-actin or total elF2 and PERK. Data are shown as mean \pm SEM, where each measurement was performed on 3 independent days on 6 donors/group. $*$ denotes $p$ value $<0.05$, when comparing both treatments connected by a line, determined by 2-way ANOVA with Tukey's post hoc test (>2 groups).

enhanced the UPR in animals also infected with RSV compared to infected animals exposed to room air (RA) (Figure 4(d)). Therefore, the lungs of smokers and COPD subjects are likely to be more sensitive to viral infection induced ER stress, which may impact disease progression.

3.4. Gpx-1-1- Mice Have Heightened ER Stress and Apoptosis following Cigarette Smoke Exposure. To determine whether the loss of GPx-1 expression directly influences ER stress following inhalation of cigarette smoke in vivo, we examined ER stress markers in Gpx-1 $1^{-/-}$mice and their wild type littermates exposed to cigarette smoke for 1 year. We previously demonstrated that loss of Gpx-1 expression in mice results in enhanced air space enlargement and inflammation following long-term cigarette smoke exposure [13]. Expression levels of Atf4, Xbp1, Grp78, Grp94, Edem1, and Chop were examined in $G p x-1^{-/-}$and wild type mice. Long-term exposure to cigarette smoke did not significantly enhance ER stress marker expression in the lungs of wild type mice (Figure 5(a)). We previously observed similar findings in wild type mice [5]. However, Gpx-1 $1^{-/-}$mice exposed to cigarette smoke had enhanced gene expression of Atf4, Xbp1, Grp78, Grp94, Edem1, and Chop (Figure 5(a)). Equally, loss of Gpx1 expression resulted in elevated lung tissue protein levels of ATF4, XBP1, GRP78, GRP94, EDEM, and CHOP following smoke exposure (Figure 5(b)). Densitometry analysis confirmed significant increases in lung levels of ATF4, XBP1, GRP78, GRP94, EDEM, and CHOP in Gpx-1 ${ }^{-/-}$mice exposed to cigarette smoke (Figure 5(b)).
Prolonged activation of CHOP by ER stress can result in cellular apoptosis. Increased structural and immune cell apoptosis is also observed in COPD lungs [27]. Therefore, we examined whether $G p x-1^{-/-}$mice exposed to cigarette smoke had elevated apoptosis. Gpx-1 $1^{-/-}$mice exposed to cigarette smoke had enhanced lung cell apoptosis, observed by TUNEL, caspase-3 cleavage, and lactate dehydrogenase $(\mathrm{LDH})$ release assays (Figure 6). Gpx-1 $1^{-/-}$mice exposed to cigarette smoke exhibited the highest frequency of TUNEL positive cells (Figure 6(a)). Enhanced caspase-3 cleavage was observed in $G p x-1^{-/-}$mice exposed to cigarette smoke (Figure 6(b)). Additionally, elevated levels of $\mathrm{LDH}$ were observed in the BALF of $G p x-1^{-/-}$mice exposed to cigarette smoke compared to the other groups (Figure 6(c)), which indicates enhanced cell membrane damage in the lung. Therefore, enhanced apoptosis in the lungs could contribute to lung remodelling and failure to clear apoptotic cells could contribute to lung inflammation.

3.5. Triggering the UPR Enhances GPx-1, GPx-2, and GPx-4 Expression in Mouse Precision-Cut Lung Slices. In Gpx-1 and $G p x-2$ double knockout mice, apoptotic cells are increased in ileal crypts [28], suggesting that GPx proteins regulate apoptosis. However, the effect of ER stress on GPx proteins has not been directly investigated. To determine the effect of ER stress on GPx-1 expression, mouse precision-cut lung slices (PCLS) were exposed to the ER stress inducer, tunicamycin, for 24 hours. The concentration of tunicamycin tested did not induce LDH release from the PCLS (Figure 7(a)), indicating 


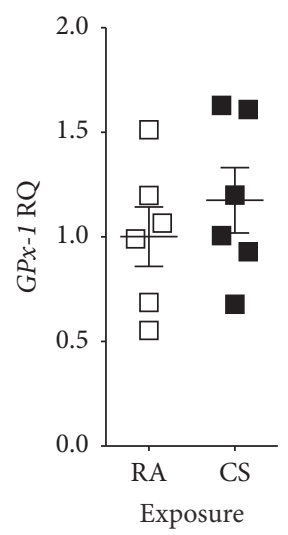

(a)

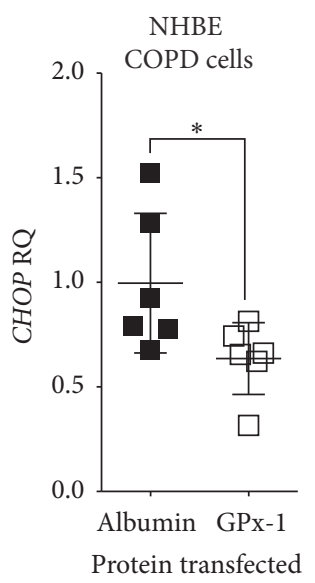

(d)

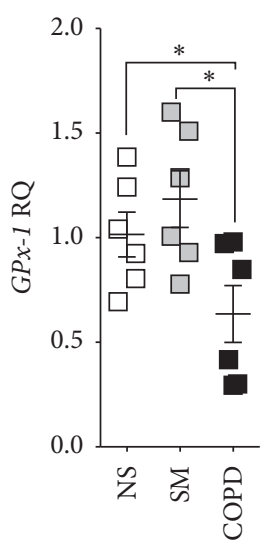

Cell source

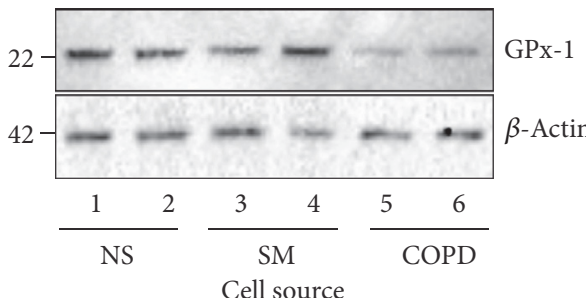

Cell source

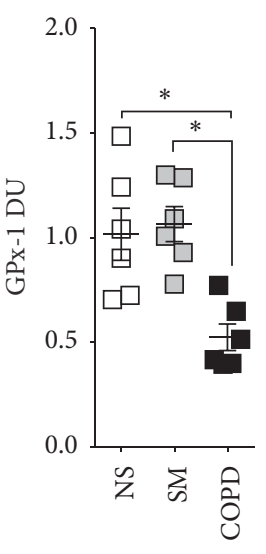

Cell source

(c)

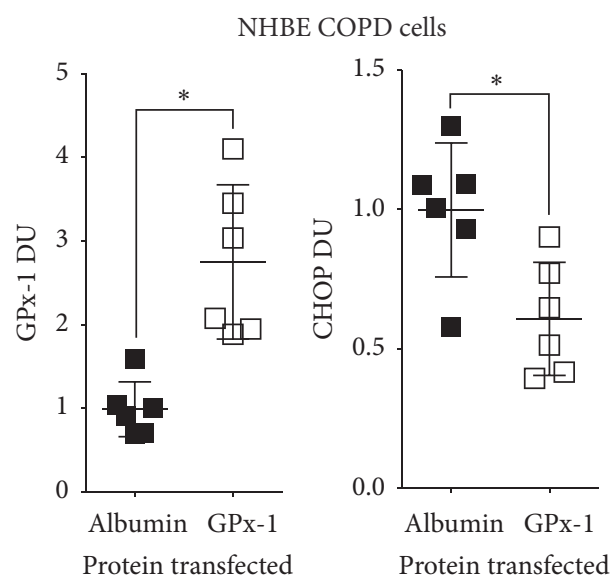

(e)

FIgURE 3: Reintroducing GPx-1 into NHBE cells isolated from COPD donors subdues the UPR. (a) Gene expression of GPx-1 was determined in fully differentiated NHBE cells from nonsmoking individuals $(n=6)$ exposed to room air (RA) and cigarette smoke (CS; from 4 cigarettes every second day (3 exposures)) using a Vitrocell VC-10 smoking robot (b) RNA and (c) protein was analyzed for GPx-1 expression from fully differentiated NHBE cells from nonsmoker (NS), smokers, (SM) and COPD (COPD) individuals ( $n=6$ donors per group). (d) NHBE cells isolated from COPD subjects were transfected with albumin or GPx-1 protein and CHOP expression was determined by qPCR. (e) Immunoblots and corresponding densitometry analysis for CHOP, Gpx-1, and $\beta$-actin from NHBE cells from COPD subjects following albumin or GPx-1 protein transfection. In each immunoblot, every lane represents an individual cell donor. Data are shown as mean \pm SEM, where each measurement was performed on 3 independent days on 6 donors/group. $*$ denotes a $p$ value $<0.05$, when comparing both treatments connected by a line, determined by Student's $t$-test (2 groups) or 2-way ANOVA with Tukey's post hoc test ( $>2$ groups).

no induction of apoptosis. Tunicamycin induced all three branches of the UPR and CHOP expression was observed in PCLS (Figures 7(b)-7(c)). Tunicamycin enhanced gene (Figure $7(\mathrm{~b})$ ) and protein (Figure $7(\mathrm{c})$ ) expression of ATF4, XBP1, ATF6, and CHOP in PCLS. Interestingly, tunicamycin induced GPx-1, GPx-2, and GPx-4 gene (Figure $7(\mathrm{~d})$ ) and protein (Figure 7(e)) expression in PCLS. Therefore, an acute ER stress induces the expression of antioxidants to counter further oxidant and ER stress.

\section{Discussion}

Cigarette smoking is the most relevant environmental risk factor associated with the development of COPD. However, smoke inhalation studies are problematic as long-term smoke exposure is required to trigger disease formation in animal models and a secondary event may be required to mimic the human disease-state. Here we observed that the loss of GPx1 expression enhances cigarette smoke-induced ER stress. GPX-1 regulates the UPR following smoke exposure and we found that the expression of GPx-1 itself was triggered by an acute ER stress stimulus. NHBE cells isolated from COPD donors expressed significantly less GPx-1, which coincides with elevated UPR. Reintroducing GPx-1 protein into NHBE cells isolated from COPD donors reduced the UPR. RSV infection contributes to loss of lung GPx-1 expression, which is exaggerated in lungs exposed to smoke and coincides with elevated UPR. Gpx-1 ${ }^{-/-}$mice exhibited greater UPR and subsequent enhanced apoptosis following long-term cigarette smoke exposure. Interestingly, triggering an acute ER stress in 


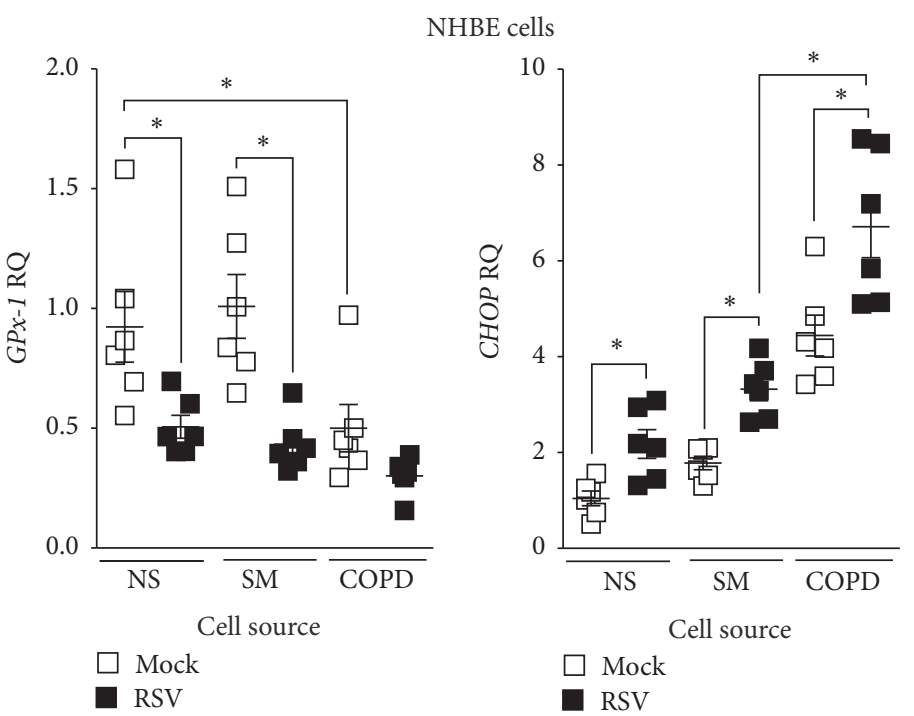

(a)

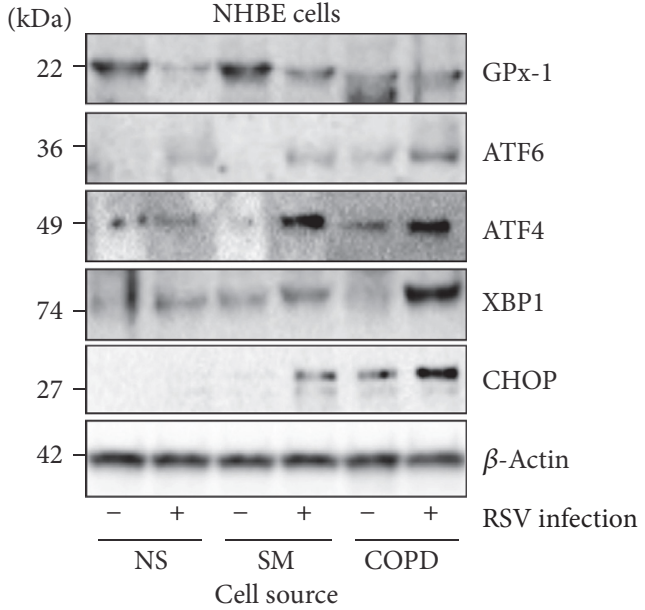

(b)

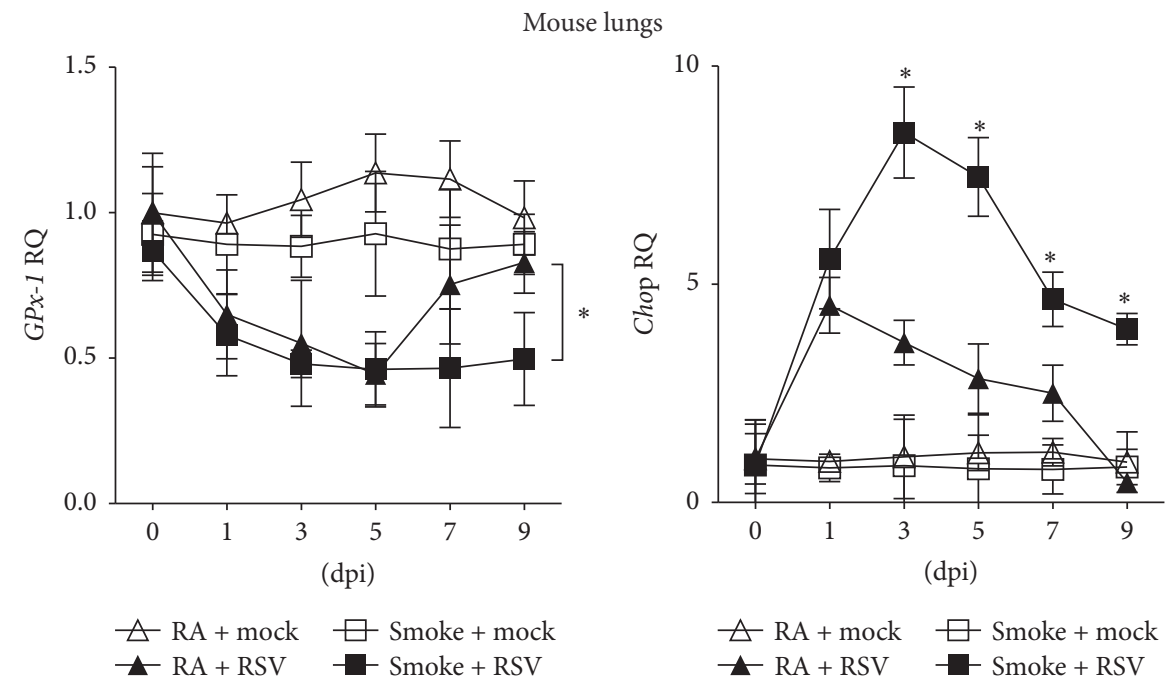

(c)

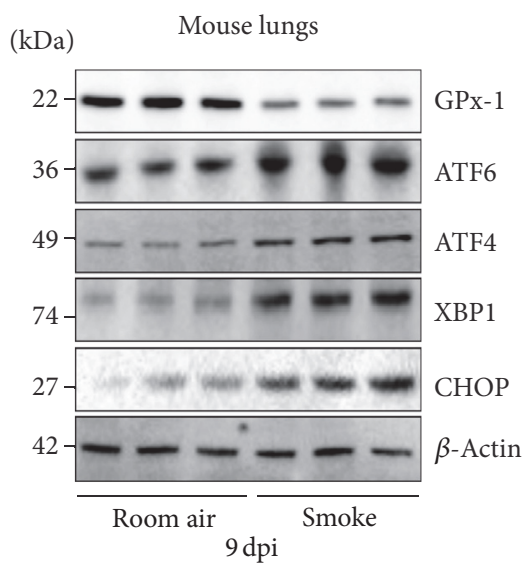

(d)

FIGURE 4: RSV infection enhances the UPR in the lung. (a) GPX-1 and CHOP gene expression were determined in NHBE cells isolated from nonsmoker (NS), smokers (SM), and COPD (COPD) individuals ( $n=6$ donors per group) infected with mock or RSV and analyzed by qPCR. (b) Protein expression of GPx-1, ATF6, ATF4, CHOP, and $\beta$-actin was examined by immunoblots. (c) Wild type mice were exposed to cigarette smoke or room air for six months and subsequently infected with $1 \times 10^{6} \mathrm{pfu}$ of RSV. Animals were euthanized at $0,1,3,5,7$, and 9 days after infection (dpi) and Gpx-1 and Chop expression were determined by qPCR. (d) Protein expression of GPx-1, ATF6, ATF4, CHOP, and $\beta$-actin was examined by immunoblots. Data are shown as mean \pm SEM, where each measurement was performed on 3 independent days. * denotes a $p$ value $<0.05$, when comparing both treatments connected by a line or the same infection day, determined by Student's $t$-test (2 groups) or 2-way ANOVA with Tukey's post hoc test ( $>2$ groups).

the lungs of mice induces a potent antioxidant response. This antioxidant response is diminished in the COPD lungs [29], which may explain the heightened UPR observed in NHBE cells isolated from COPD subjects. The exact role of this heightened UPR on the progression of COPD still remains to be fully determined. However, we have established that loss of GPx-1 in vivo leads to a marked increased in all three branches of the UPR (see Figure 8 for proposed signaling scheme) and this coincides with enhanced apoptosis and lung tissue destruction in mice [13]. Our results suggest that GPx1 significantly regulates the UPR in COPD and enhancing
GPx-1 expression may be feasible means of offsetting the UPR and lung injury responses that drive the onset and progression of this disease.

Multiple studies utilizing the $G p x-1^{-/-}$and transgenic mice demonstrated the protective role of GPx-1 in countering oxidative injury and cell death mediated by ROS $[13,30]$. GPX-1 activity also affects protein kinase phosphorylation [31] and oxidant-mediated activation of NF- $\kappa \mathrm{B}$ [32]. In this current study, GPx-1 was significantly reduced in NHBE cells isolated from COPD subjects compared to nonsmokers and smokers. Others have reported that the alteration of GPx-1 


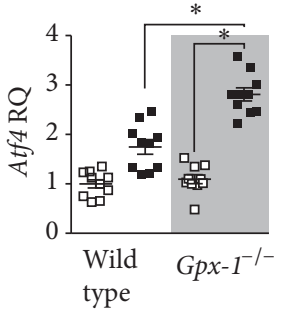

$\square$ Room air
$\square$ Cigarette smoke

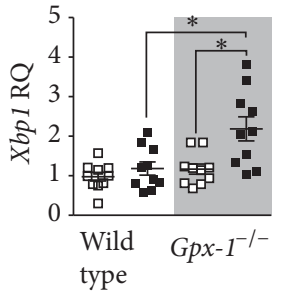

$\square$ Room air

- Cigarette smoke
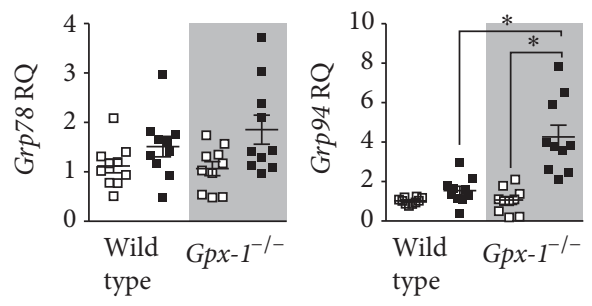

$\square$ Room air

Cigarette smoke $\square$ Room air

Cigarette smoke
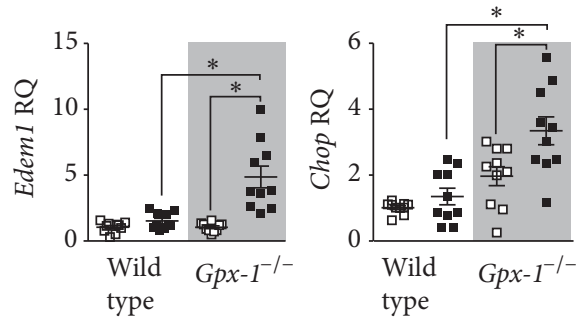

$\square$ Room air

- Cigarette smoke $\square$ Room air

- Cigarette smoke

(a)

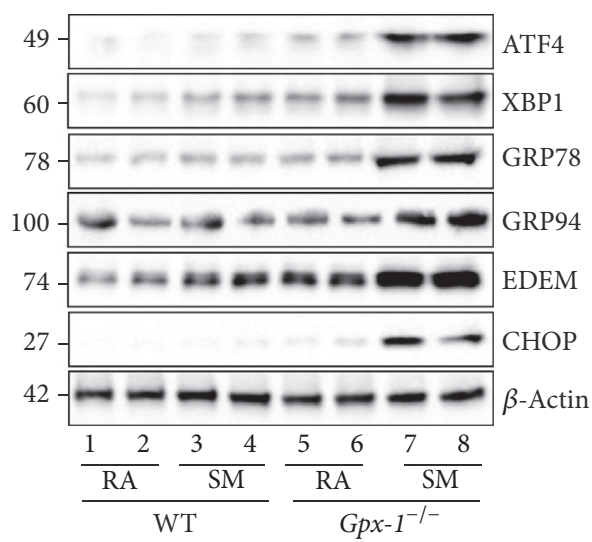

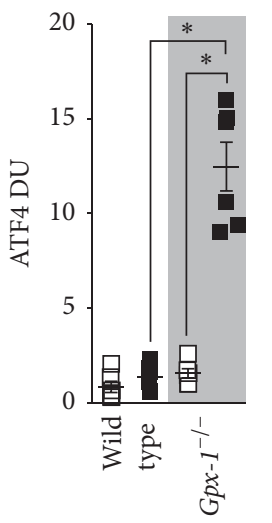

Room air

Cigarette smoke

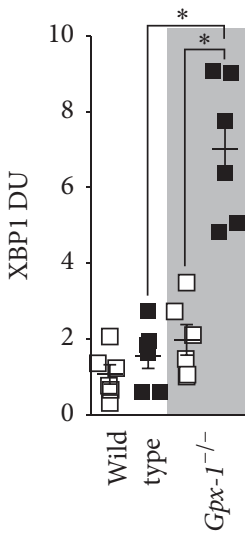

$\square$ Room air

Cigarette smoke

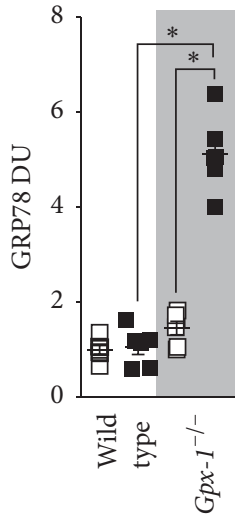

$\square$ Room air

Cigarette smoke

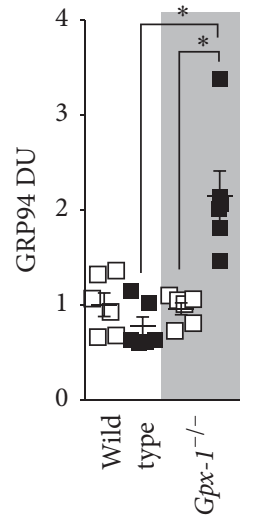

$\square$ Room air

Cigarette smoke

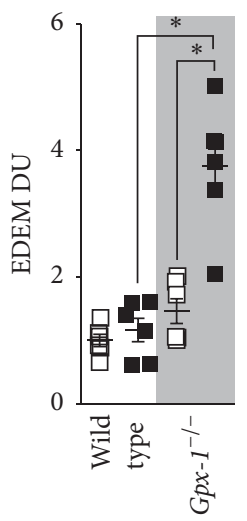

$\square$ Room air

Cigarette smoke

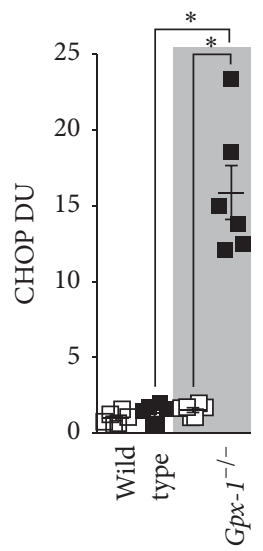

$\square$ Room air

Cigarette smoke

(b)

FIGURE 5: Gpx-1 deficient mice have heightened ER stress in their lungs following exposure to cigarette smoke. Gpx-1 ${ }^{-1-}$ and wild type mice were exposed to cigarette smoke daily for 1 year. (a) Lung gene expression of Chop, Atf4, Edem1, Grp78, Grp94, and Xbp1 was examined. (b) Immunoblots were performed of whole lung protein for CHOP, ATF4, EDEM, GRP78, GRP94, and XBP1. Dot plots are represented as (a) relative quantification (RQ) compared to ACTB expression or (b) densitometry units (DU) of pixel intensity expressed as a ratio to $\beta$-actin. Every lane represents an individual mouse. Data are shown as mean \pm SEM, where each measurement was performed on 3 independent days on 6 donors/group. $*$ denotes a $p$ value $<0.05$, when comparing both treatments connected by a line, determined by 2 -way ANOVA with Tukey's post hoc test (>2 groups).

expression does not affect the mRNA or activity expression of other selenoproteins [33], which suggests no compensation expression of other selenoproteins following loss of GPx1 expression. Currently the mechanism by which cigarette smoke regulates GPx-1 expression is not fully elucidated but considering our $\mathrm{GPx}-1$ reintroduction data further analysis of GPx-1 regulation is critical. GPx-1 expression and activity have been reported to be regulated by Nrf2 [34], the transcription factor TFAP2C [35], CpG methylation of the GPx-1 promoter [35], Bcr-Abl/mTOR [36], selenium [37], estrogen [38], adenosine [39], Sec-insertion sequence (SECIS) factors [40], EGFR [41], and homocysteine [42]. Specifically, within 


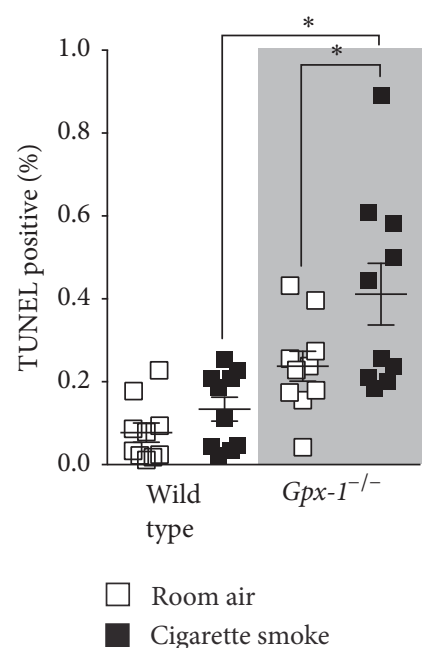

(a)

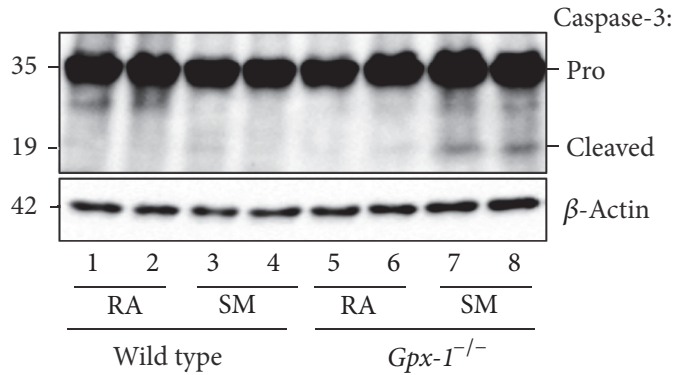

(b)

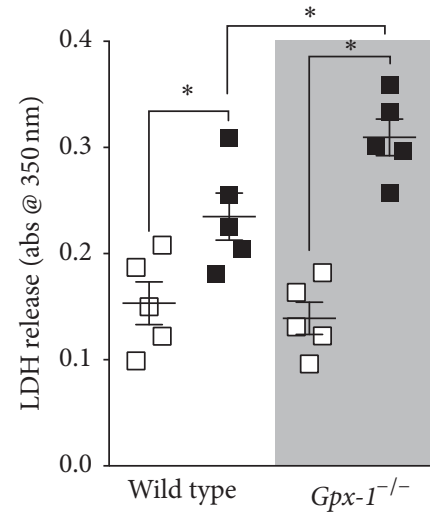

(c)

FIGURE 6: Gpx-1 deficient mice have heightened ER stress in their lungs following exposure to cigarette smoke. Gpx-1 $1^{-/-}$and wild type mice were exposed to cigarette smoke daily for 1 year. (a) TUNEL analysis was performed on lung tissue from each mouse group. (b) Enhanced lung tissue caspase-3 cleavage coincided with (c) elevated LDH into BALF of $G p x-1^{-1-}$ exposed to cigarette smoke. Every lane in (b) represents an individual mouse and densitometry units (DU) of pixel intensity expressed as a ratio to total caspase-3 levels. Dot plots are represented as mean \pm SEM, where each measurement was performed on 3 independent days on 6 donors/group. $*$ denotes a $p$ value $<0.05$, when comparing both treatments connected by a line, determined by 2-way ANOVA with Tukey’s post hoc test ( $>2$ groups).

the lung during smoke exposure, Singh et al. show elevated GPX-1 expression in the lungs following one-month cigarette smoke exposure that was regulated by Nrf2 [34]. However, Nrf2 expression is lost in COPD subjects suggesting that this secondary event could result in reduced GPx-1 expression and heightened ER stress. Loss of $\mathrm{Nrf2}$ in mice results in enhanced susceptibility to cigarette smoke $[43,44]$ and elastase [45] induced emphysema in mice. However genomic studies in $\mathrm{Nrf2}^{-/-}$mouse samples suggest that Nrf2 may regulate other GPx genes but not GPx-1 [46]. Whether RSV infection alters regulation of GPx-1 expression in a similar manner to chronic smoke is unknown. Interestingly, the UPR upon RSV infection can counter viral proliferation [26]. Further studies on the regulation of GPx-1 in smoke exposure and COPD and the significance of ER stress in the lungs are required. This will be a major area for our future work.
Since GPx-1 expression regulates all three branches of the UPR, GPx-1 may affect a common mediator of the UPR or each branch individually. Dissociation of GRP78/BiP upon ER stress is required for all three branches of the UPR. GPx-1 expression directly regulated the gene expression of GRP78. However, whether GPx-1 impacts on GRP78 dissociation during ER stress is unknown. Nrf2 interacts directly with PERK [47] and may play a major role in GPx-1 expression thereby regulating the UPR. The ER stress inducer, thapsigargin, induces Nrf2 protein production in 16-HBE cells [47], which suggests that the UPR induces a Nrf2 response to reverse ER stress. We observe a similar effect on GPx-1 expression in PCLS following tunicamycin treatment. Equally, XBP1 regulates several antioxidants, including catalase, SOD1, and thioredoxin TRX1 [48]. However, XBP1 does not regulate GPx proteins [48] but XBP1 expression is regulated by GPx-1. The 


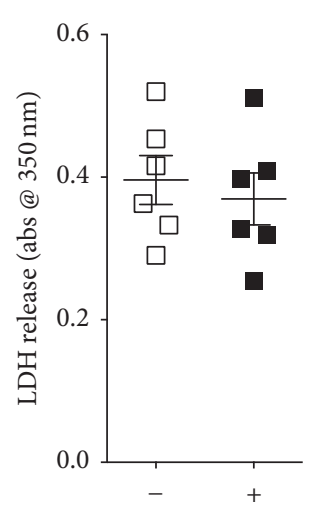

Tunicamycin $(1 \mu \mathrm{M})$

(a)

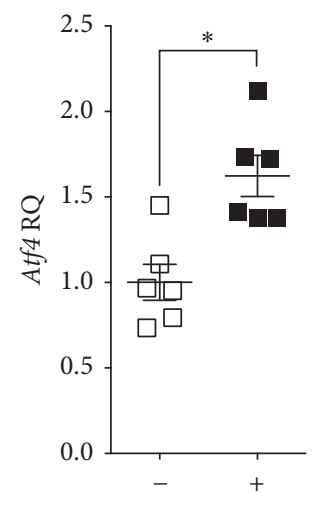

Tunicamycin $(1 \mu \mathrm{M})$

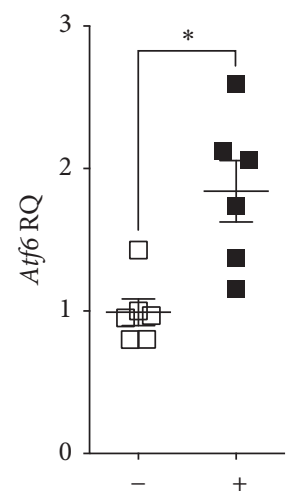

Tunicamycin $(1 \mu \mathrm{M})$

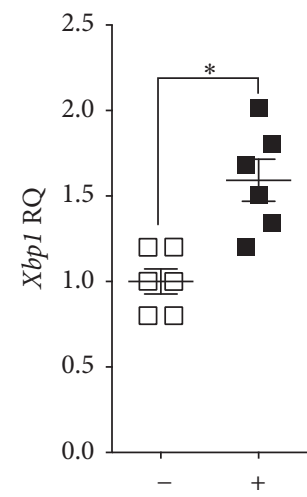

Tunicamycin $(1 \mu \mathrm{M})$

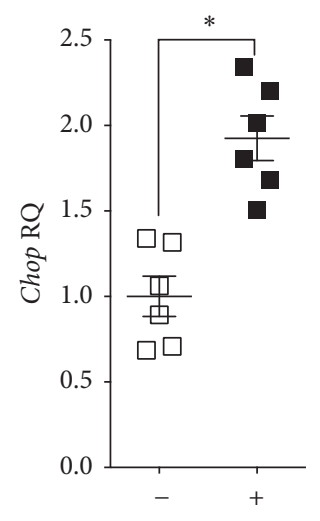

Tunicamycin $(1 \mu \mathrm{M})$

(b)

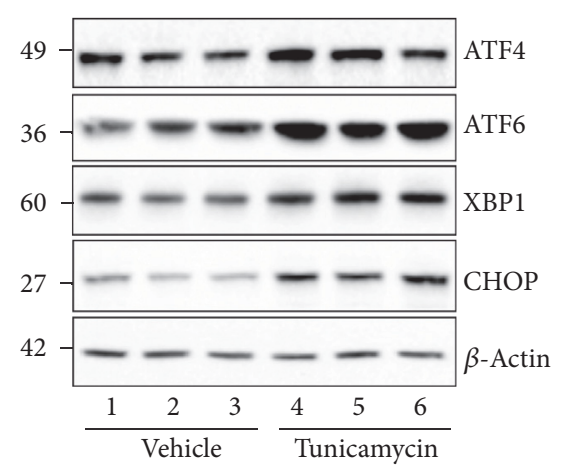

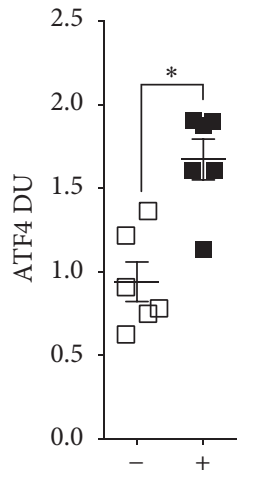

Tunicamycin $(1 \mu \mathrm{M})$
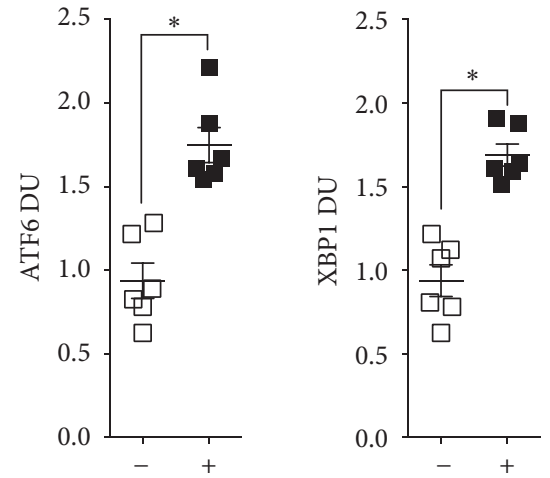

Tunicamycin $(1 \mu \mathrm{M}) \quad$ Tunicamycin $(1 \mu \mathrm{M})$

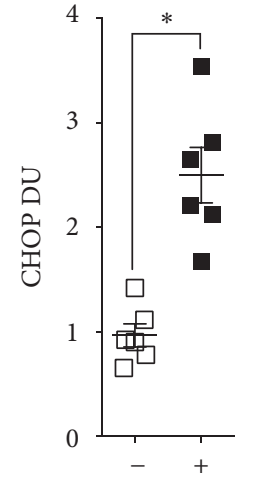

Tunicamycin $(1 \mu \mathrm{M})$

(c)
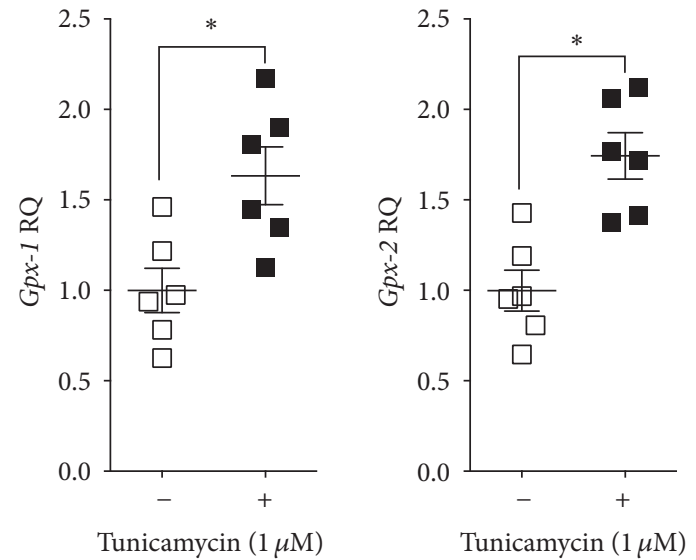

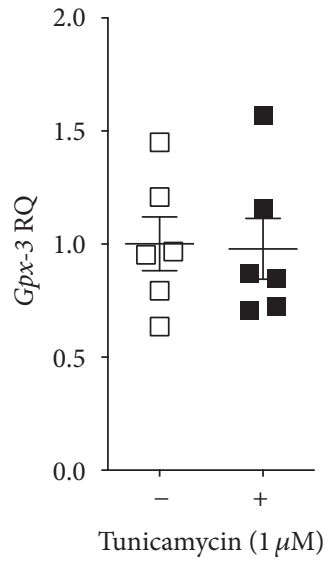

(d)
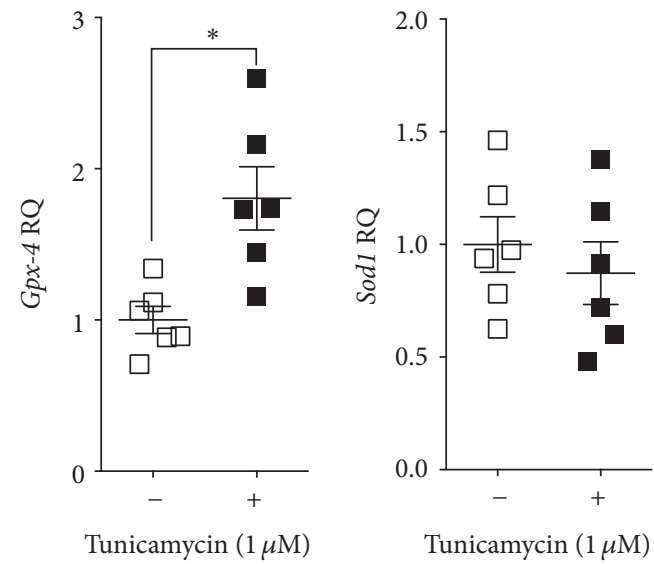

Figure 7: Continued. 


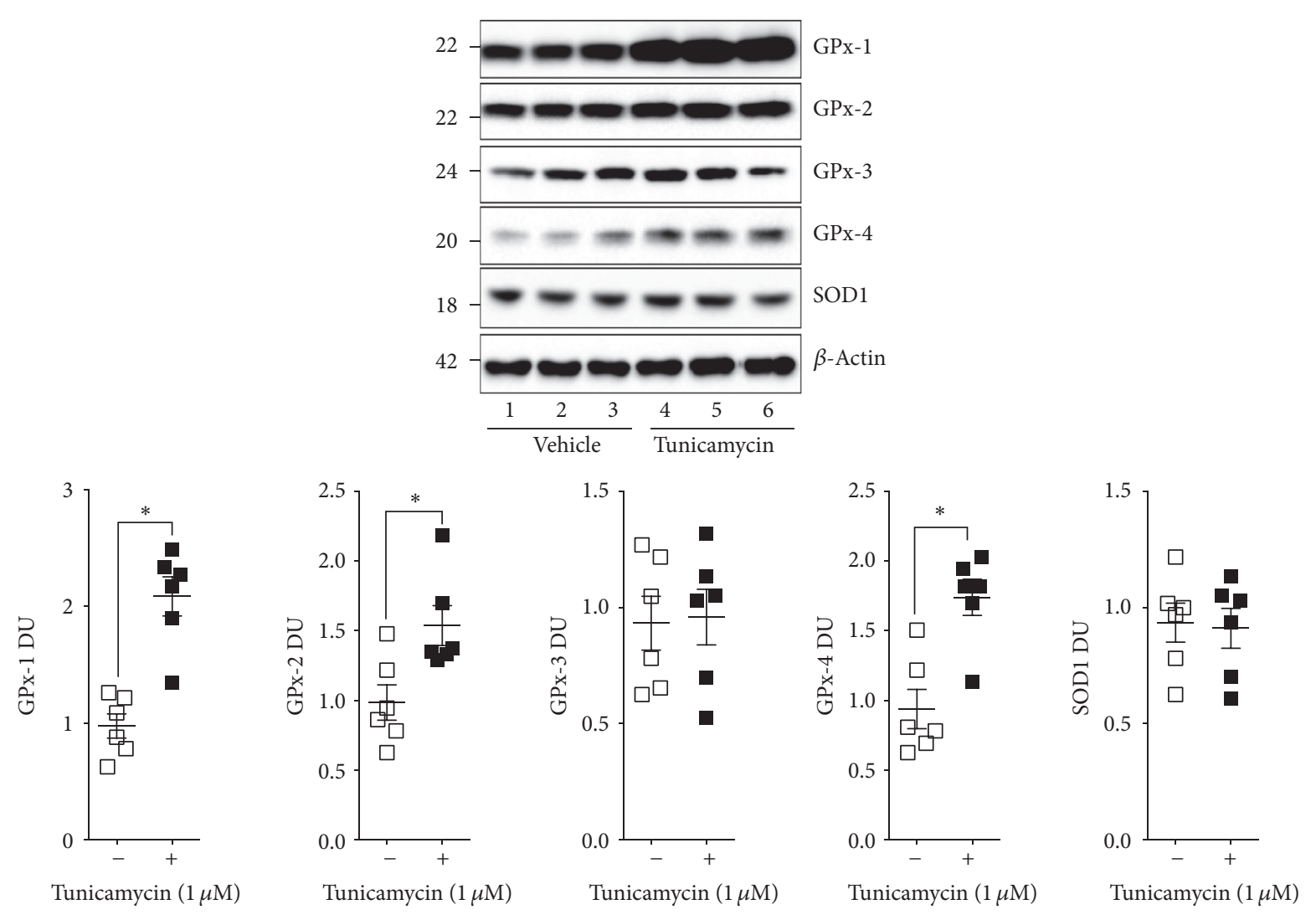

(e)

FIGURE 7: Effect of ER stress-induced tunicamycin on the antioxidant expression profile in mouse precision-cut lung slices (PCLS). PCLS were obtained from wild type mice and were exposed to tunicamycin $(1 \mu \mathrm{M})$ for 24 hours. (a) LDH release into media and (b) Chop, Atf4, Atf6, and Xbpl gene expression were examined. (c) Immunoblots were conducted for Chop, Atf4, Atf6, Xbpl, and $\beta$-actin. (d) GPx-1, GPx-2, $G P x-3, G P x-4$, and Sod1 were quantified by qPCR and (e) immunoblots analysis. Every lane represents an individual mouse. Dot plots are represented as relative quantification (RQ) compared to ACTB expression or densitometry units (DU) of pixel intensity expressed as a ratio to $\beta$-actin. Data are shown as the mean \pm SEM, where each measurement was performed on 3 independent days on 6 donors/group. $*$ denotes a $p$ value $<0.05$, when comparing both treatments connected by a line, determined by Student's $t$-test ( 2 groups).

$\mathrm{XBP1}$ regulated gene, EDEM1, was also enhanced in $G p x-1^{-1-}$ mice exposed to smoke, which further confirms that GPx-1 regulation of XBP1 signaling. ATF6 requires translocation to the Golgi to undergo cleavage and subsequent translocation to the nucleus to act as a transcription factor [49]. Whether GPX-1 modulates this signaling has yet to be determined. This crosstalk between antioxidant signaling and the UPR is partially lost in COPD and may play a critical step in the pathogenesis of this disease.

The role of the UPR on apoptosis is dependent on the stimulus, exposure duration, and intensity of this signaling. Loss of GPX-1 expression directly impacts cell death and cell death is an important factor in COPD progression [50]. Smoke-induced apoptosis has been associated with several processes, such as ceramide signaling [51], damage-associated molecular pattern molecules (DAMPs) [52], and tumor necrosis factor-related apoptosis-inducing ligand (TRAIL) [53]. The loss of Gpx-1 exacerbated cigarette smoke-induced cell apoptosis in mice, suggesting that Gpx-1/- genotype exacerbated cell death at least partially through the induction of the UPR. Our group has previously demonstrated that $\mathrm{GPX}-1$ also regulates the activation of protein tyrosine phosphatase 1B (PTP1B) and protein phosphatase 2A (PP2A) [13]. Both of these phosphatases could impact smoke-induced cell survival $[54,55]$. Equally, Nrf2 deficient cells undergo enhanced cell death following exposure to ER stress [47], which may be dependent on GPx-1 expression. Therefore, the data presented here suggests that enhanced $\mathrm{CHOP}$ expression in NHBE cells and mouse lungs may contribute to apoptosis. Other studies also suggest that certain elements of the UPR have several antiapoptotic and anti-inflammatory effects in other organs. XBP1 reduces CSE-induced CHOP and thereby is protected from CSE-induced apoptosis in a retinal pigment epithelia (RPE) cell line [56], via regulation of eIF2 $\alpha$ and p38 phosphorylation [4]. Loss of CHOP expression exacerbated cell death through the downregulation of Nrf2 in RPE cells [4]. CHOP deficiency enhances apoptosis in hippocampal cells and impaired memory-related behavioural performances in mice with tunicamycin treatment [57]. Recently, deficiency of CHOP exaggerated lipopolysaccharide- (LPS-) induced inflammation and kidney injury in mice [58]. The importance of ER stress and the role of each member of 


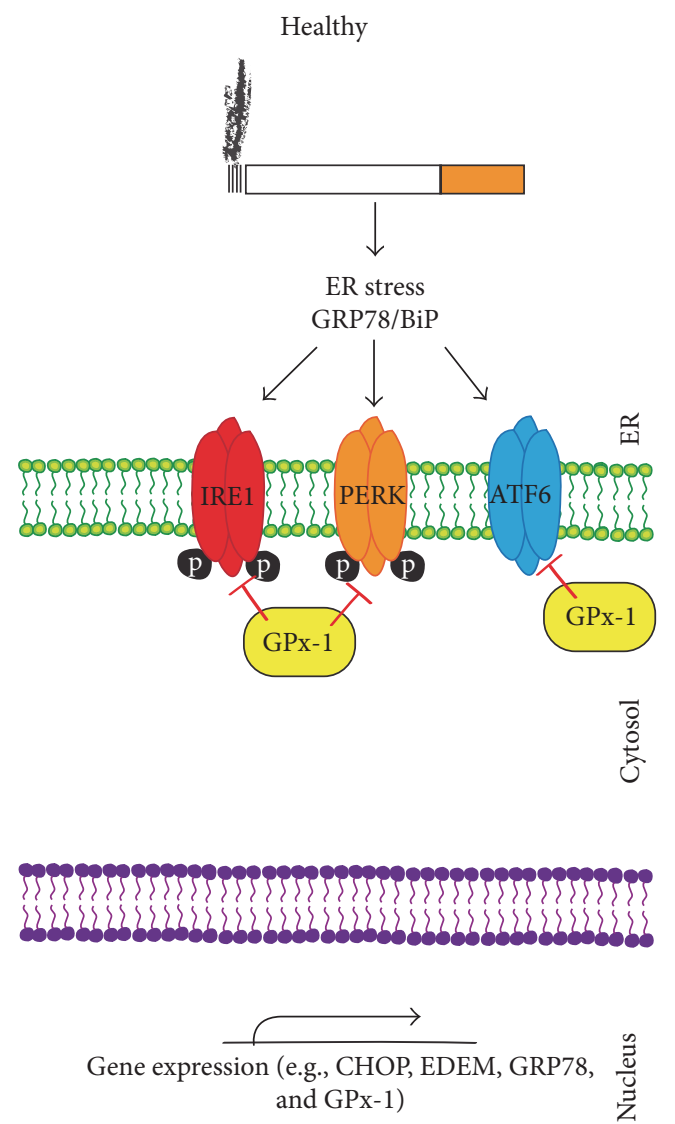

(a)

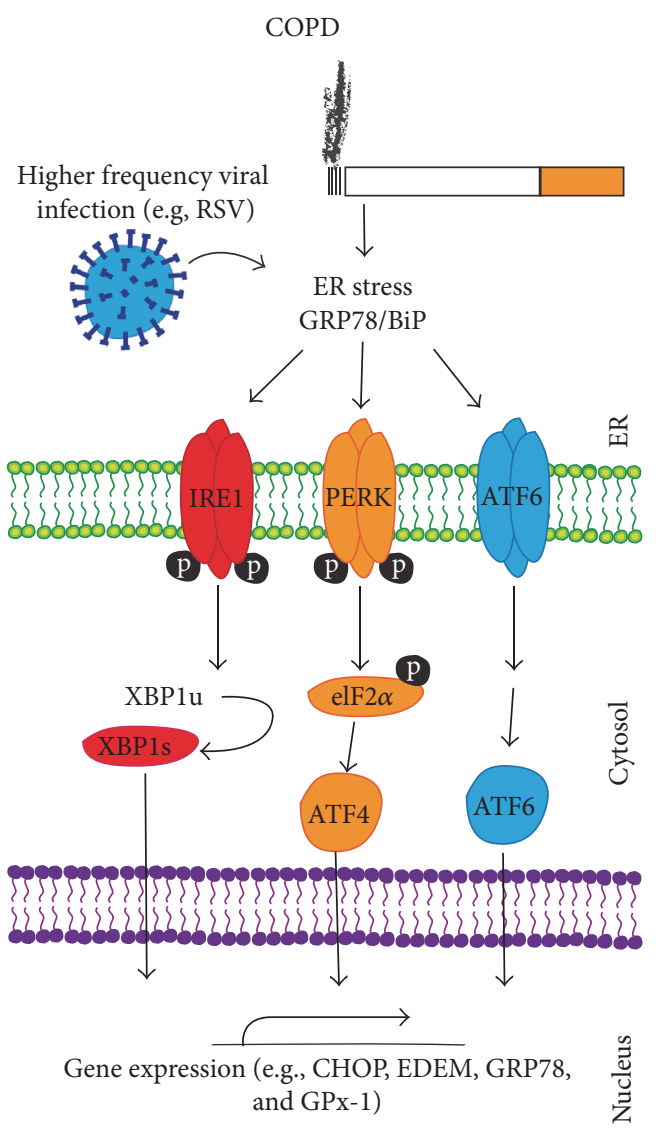

(b)

FIGURE 8: Possible signaling mechanism for GPx-1 regulation of the UPR. Evidence presented in this study indicates that following smoke exposure GPx-1 prevents ER stress (a). However, in the disease-state GPx-1 expression is subdued and results in enhanced UPR (b). RSV infection significantly contributes to reduced GPx-1 expression that coincides with enhanced UPR.

the UPR in the development of lung disease still remain to be fully addressed. However, we have demonstrated that enhanced UPR coincides with worsening of symptoms that are countered with the expression of GPx-1 in mice.

\section{Conclusion}

Here we demonstrate that GPx-1 expression is reduced in NHBE cells isolated from COPD subjects, GPx-1 is a major regulator of the UPR under smoke exposure conditions, and acute ER stress induces lung GPx-1 expression. Together, our data indicate that the loss of GPX-1 expression in COPD lungs could contribute to disease progression by enhancing the UPR. These studies suggest that enhancing GPX-1 activity may be an effective therapeutic approach to prevent the damage induced by UPR in the lung.

\section{Competing Interests}

None of the authors have a financial relationship with a commercial entity that has an interest in the subject of this manuscript.

\section{Acknowledgments}

This work was supported by grants made available to Patrick Geraghty (Flight Attendant Medical Research Institute (YCSA 113380)), Robert F. Foronjy (US National Institutes of Health 5R01HL098528-05 and Flight Attendant Medical Research Institute (CIA 130020)), Matthias A. Salathe (Flight Attendant Medical Research Institute CIA 103027 and 130033; the James and Esther King Biomedical Research Program of the State of FL 5JK02), and Jeanine M. D'Armiento (R01 HL086936-07).

\section{References}

[1] J. Xu, S. L. Murphy, K. D. Kochanek, and B. A. Basstian, "Deaths: final data for 2013," in National Vital Statistics Reports, pp. 1119, National Center for Health Statistics, Hyattsville, Md, USA, 2016.

[2] R. F. Foronjy, O. Mirochnitchenko, O. Propokenko et al., "Superoxide dismutase expression attenuates cigarette smokeor elastase-generated emphysema in mice," American Journal of Respiratory and Critical Care Medicine, vol. 173, no. 6, pp. 623631, 2006. 
[3] E. Bargagli, C. Olivieri, D. Bennett, A. Prasse, J. MullerQuernheim, and P. Rottoli, "Oxidative stress in the pathogenesis of diffuse lung diseases: a review," Respiratory Medicine, vol. 103, no. 9, pp. 1245-1256, 2009.

[4] C. Huang, J. J. Wang, J. H. Ma, C. Jin, Q. Yu, and S. X. Zhang, "Activation of the UPR protects against cigarette smokeinduced RPE apoptosis through up-regulation of Nrf2," Journal of Biological Chemistry, vol. 290, no. 9, pp. 5367-5380, 2015.

[5] P. Geraghty, A. Wallace, and J. M. D'Armiento, "Induction of the unfolded protein response by cigarette smoke is primarily an activating transcription factor 4-C/EBP homologous protein mediated process," International Journal of Chronic Obstructive Pulmonary Disease, vol. 6, no. 1, pp. 309-319, 2011.

[6] A. Hengstermann and T. Müller, "Endoplasmic reticulum stress induced by aqueous extracts of cigarette smoke in 3T3 cells activates the unfolded-protein-response-dependent PERK pathway of cell survival," Free Radical Biology and Medicine, vol. 44, no. 6, pp. 1097-1107, 2008.

[7] E. Jorgensen, A. Stinson, L. Shan, J. Yang, D. Gietl, and A. P. Albino, "Cigarette smoke induces endoplasmic reticulum stress and the unfolded protein response in normal and malignant human lung cells," BMC Cancer, vol. 8, article 229, 2008.

[8] S. G. Kelsen, X. Duan, R. Ji, O. Perez, C. Liu, and S. Merali, "Cigarette smoke induces an unfolded protein response in the human lung: a proteomic approach," American Journal of Respiratory Cell and Molecular Biology, vol. 38, no. 5, pp. 541550, 2008.

[9] Y. Tagawa, N. Hiramatsu, A. Kasai et al., "Induction of apoptosis by cigarette smoke via ROS-dependent endoplasmic reticulum stress and CCAAT/enhancer-binding protein-homologous protein (CHOP)," Free Radical Biology and Medicine, vol. 45, no. 1, pp. 50-59, 2008.

[10] R. J. Kaufman, "Stress signaling from the lumen of the endoplasmic reticulum: coordination of gene transcriptional and translational controls," Genes \& Development, vol. 13, no. 10, pp. 1211-1233, 1999.

[11] P. Walter and D. Ron, "The unfolded protein response stress pathway to homeostatic regulation," Science, vol. 334, no. 6059, pp. 1081-1086, 2011.

[12] A.-H. Lee, N. N. Iwakoshi, and L. H. Glimcher, "XBP-1 regulates a subset of endoplasmic reticulum resident chaperone genes in the unfolded protein response," Molecular and Cellular Biology, vol. 23, no. 21, pp. 7448-7459, 2003.

[13] P. Geraghty, A. A. Hardigan, A. M. Wallace et al., "The glutathione peroxidase 1-protein tyrosine phosphatase $1 \mathrm{~B}$-protein phosphatase 2A axis. A key determinant of airway inflammation and alveolar destruction," American Journal of Respiratory Cell and Molecular Biology, vol. 49, no. 5, pp. 721-730, 2013.

[14] M. A. Forgione, N. Weiss, S. Heydrick et al., "Cellular glutathione peroxidase deficiency and endothelial dysfunction," American Journal of Physiology-Heart and Circulatory Physiology, vol. 282, no. 4, pp. H1255-H1261, 2002.

[15] J. B. De Haan, C. Bladier, M. Lotfi-Miri et al., "Fibroblasts derived from Gpx1 knockout mice display senescent-like features and are susceptible to $\mathrm{H} 2 \mathrm{O} 2$-mediated cell death," Free Radical Biology and Medicine, vol. 36, no. 1, pp. 53-64, 2004.

[16] P. Lewis, N. Stefanovic, J. Pete et al., "Lack of the antioxidant enzyme glutathione peroxidase- 1 accelerates atherosclerosis in diabetic apolipoprotein E-deficient mice," Circulation, vol. 115, no. 16, pp. 2178-2187, 2007.

[17] C. Duong, H. J. Seow, S. Bozinovski, P. J. Crack, G. P. Anderson, and R. Vlahos, "Glutathione peroxidase-1 protects against cigarette smoke-induced lung inflammation in mice," American Journal of Physiology_Lung Cellular and Molecular Physiology, vol. 299, no. 3, pp. L425-L433, 2010.

[18] M.-C. Nlend, R. J. Bookman, G. E. Conner, and M. Salathe, "Regulator of G-protein signaling protein 2 modulates purinergic calcium and ciliary beat frequency responses in airway epithelia," American Journal of Respiratory Cell and Molecular Biology, vol. 27, no. 4, pp. 436-445, 2002.

[19] A. M. Wallace, A. Hardigan, P. Geraghty et al., "Protein phosphatase $2 \mathrm{~A}$ regulates innate immune and proteolytic responses to cigarette smoke exposure in the lung," Toxicological Sciences, vol. 126, no. 2, pp. 589-599, 2012.

[20] R. F. Foronjy, M. A. Salathe, A. J. Dabo et al., "TLR9 expression is required for the development of cigarette smoke-induced emphysema in mice," American Journal of Physiology-Lung Cellular and Molecular Physiology, vol. 311, no. 1, pp. L154-L166, 2016.

[21] A. R. Ressmeyer, A. K. Larsson, E. Vollmer, S. E. Dahlèn, S. Uhlig, and C. Martin, "Characterisation of guinea pig precisioncut lung slices: comparison with human tissues," European Respiratory Journal, vol. 28, no. 3, pp. 603-611, 2006.

[22] H.-D. Held, C. Martin, and S. Uhlig, "Characterization of airway and vascular responses in murine lungs," British Journal of Pharmacology, vol. 126, no. 5, pp. 1191-1199, 1999.

[23] C. Chen, J. J. Wang, J. Li, Q. Yu, and S. X. Zhang, "Quinotrierixin inhibits proliferation of human retinal pigment epithelial cells," Molecular Vision, vol. 19, pp. 39-46, 2013.

[24] J. A. Wedzicha, "Airway infection accelerates decline of lung function in chronic obstructive pulmonary disease," American Journal of Respiratory and Critical Care Medicine, vol. 164, no. 10, pp. 1757-1758, 2001.

[25] P. Mallia and S. L. Johnston, "Mechanisms and experimental models of chronic obstructive pulmonary disease exacerbations," Proceedings of the American Thoracic Society, vol. 2, no. 4, pp. 361-366, 2005.

[26] I. Hassan, K. S. Gaines, W. J. Hottel et al., "Inositol-requiring enzyme 1 inhibits respiratory syncytial virus replication," Journal of Biological Chemistry, vol. 289, no. 11, pp. 7537-7546, 2014.

[27] S. Hodge, G. Hodge, M. Holmes, and P. N. Reynolds, "Increased airway epithelial and T-cell apoptosis in COPD remains despite smoking cessation," European Respiratory Journal, vol. 25, no. 3, pp. 447-454, 2005.

[28] F.-F. Chu, R. S. Esworthy, P. G. Chu et al., "Bacteria-induced intestinal cancer in mice with disrupted Gpx1 and Gpx2 genes," Cancer Research, vol. 64, no. 3, pp. 962-968, 2004.

[29] M. Tomaki, H. Sugiura, A. Koarai et al., "Decreased expression of antioxidant enzymes and increased expression of chemokines in COPD lung," Pulmonary Pharmacology and Therapeutics, vol. 20, no. 5, pp. 596-605, 2007.

[30] W.-H. Cheng, Y.-S. Ho, B. A. Valentine, D. A. Ross, G. F. Combs Jr., and X. G. Lei, "Cellular glutathione peroxidase is the mediator of body selenium to protect against paraquat lethality in transgenic mice," Journal of Nutrition, vol. 128, no. 7, pp. 10701076, 1998.

[31] M. A. Nasr, M. J. Fedele, K. Esser, and A. M. Diamond, "GPx1 modulates AKT and P70S6K phosphorylation and Gadd45 levels in MCF-7 cells," Free Radical Biology and Medicine, vol. 37, no. 2, pp. 187-195, 2004.

[32] Q. Li, S. Sanlioglu, S. Li, T. Ritchie, L. Oberley, and J. F. Engelhardt, "GPx-1 gene delivery modulates $\mathrm{NF} \kappa \mathrm{B}$ activation following diverse environmental injuries through a specific 
subunit of the IKK complex," Antioxidants and Redox Signaling, vol. 3, no. 3, pp. 415-432, 2001.

[33] W.-H. Cheng, Y.-S. Ho, D. A. Ross, B. A. Valentine, G. F. Combs Jr., and X. G. Lei, "Cellular glutathione peroxidase knockout mice express normal levels of selenium-dependent plasma and phospholipid hydroperoxide glutathione peroxidases in various tissues," Journal of Nutrition, vol. 127, no. 8, pp. 1445-1450, 1997.

[34] A. Singh, T. Rangasamy, R. K. Thimmulappa et al., "Glutathione peroxidase 2, the major cigarette smoke-inducible isoform of GPX in lungs, is regulated by Nrf2," American Journal of Respiratory Cell and Molecular Biology, vol. 35, no. 6, pp. 639650, 2006.

[35] M. V. Kulak, A. R. Cyr, G. W. Woodfield et al., "Transcriptional regulation of the GPX1 gene by TFAP2C and aberrant CpG methylation in human breast cancer," Oncogene, vol. 32, no. 34, pp. 4043-4051, 2013.

[36] E. N. Reinke, D. N. Ekoue, S. Bera, N. Mahmud, and A. M. Diamond, "Translational regulation of GPx-1 and GPx-4 by the mTOR pathway," PLoS ONE, vol. 9, no. 4, Article ID e93472, 2014.

[37] L. Flohe, W. A. Günzler, and H. H. Schock, "Glutathione peroxidase: a selfnoenzyme," FEBS Letters, vol. 32, no. 1, pp. 132134, 1973.

[38] J. M. Lean, C. J. Jagger, B. Kirstein, K. Fuller, and T. J. Chambers, "Hydrogen peroxide is essential for estrogen-deficiency bone loss and osteoclast formation," Endocrinology, vol. 146, no. 2, pp. 728-735, 2005.

[39] Y. Zhang, D. E. Handy, and J. Loscalzo, "Adenosine-dependent induction of glutathione peroxidase 1 in human primary endothelial cells and protection against oxidative stress," Circulation Research, vol. 96, no. 8, pp. 831-837, 2005.

[40] C. B. Allan, G. M. Lacourciere, and T. C. Stadtman, "Responsiveness of selenoproteins to dietary selenium," Annual Review of Nutrition, vol. 19, pp. 1-16, 1999.

[41] C. Duval, N. Augé, M.-F. Frisach, L. Casteilla, R. Salvayre, and A. Nègre-Salvayre, "Mitochondrial oxidative stress is modulated by oleic acid via an epidermal growth factor receptor-dependent activation of glutathione peroxidase," Biochemical Journal, vol. 367, no. 3, pp. 889-894, 2002.

[42] D. E. Handy, Y. Zhang, and J. Loscalzo, "Homocysteine downregulates cellular glutathione peroxidase (GPxl) by decreasing translation," Journal of Biological Chemistry, vol. 280, no. 16, pp. 15518-15525, 2005.

[43] T. Rangasamy, C. Y. Cho, R. K. Thimmulappa et al., "Genetic ablation of Nrf2 enhances susceptibility to cigarette smokeinduced emphysema in mice," Journal of Clinical Investigation, vol. 114, no. 9, pp. 1248-1259, 2004.

[44] T. Iizuka, Y. Ishii, K. Itoh et al., "Nrf2-deficient mice are highly susceptible to cigarette smoke-induced emphysema," Genes to Cells, vol. 10, no. 12, pp. 1113-1125, 2005.

[45] Y. Ishii, K. Itoh, Y. Morishima et al., "Transcription factor Nrf2 plays a pivotal role in protection against elastaseinduced pulmonary inflammation and emphysema," Journal of Immunology, vol. 175, no. 10, pp. 6968-6975, 2005.

[46] J.-M. Lee, M. J. Calkins, K. Chan, Y. W. Kan, and J. A. Johnson, "Identification of the NF-E2-related factor-2-dependent genes conferring protection against oxidative stress in primary cortical astrocytes using oligonucleotide microarray analysis," Journal of Biological Chemistry, vol. 278, no. 14, pp. 12029-12038, 2003.

[47] S. B. Cullinan, D. Zhang, M. Hannink, E. Arvisais, R. J. Kaufman, and J. A. Diehl, "Nrf2 is a direct PERK substrate and effector of PERK-dependent cell survival," Molecular and Cellular Biology, vol. 23, no. 20, pp. 7198-7209, 2003.

[48] Y. Liu, M. Adachi, S. Zhao et al., "Preventing oxidative stress: a new role for XBP1," Cell Death and Differentiation, vol. 16, no. 6, pp. 847-857, 2009.

[49] K. Haze, H. Yoshida, H. Yanagi, T. Yura, and K. Mori, "Mammalian transcription factor ATF6 is synthesized as a transmembrane protein and activated by proteolysis in response to endoplasmic reticulum stress," Molecular Biology of the Cell, vol. 10, no. 11, pp. 3787-3799, 1999.

[50] L. Segura-Valdez, A. Pardo, M. Gaxiola, B. D. Uhal, C. Becerril, and M. Selman, "Upregulation of gelatinases A and B, collagenases 1 and 2, and increased parenchymal cell death in COPD," Chest, vol. 117, no. 3, pp. 684-694, 2000.

[51] I. Petrache, V. Natarajan, L. Zhen et al., "Ceramide upregulation causes pulmonary cell apoptosis and emphysema-like disease in mice," Nature Medicine, vol. 11, no. 5, pp. 491-498, 2005.

[52] R. F. Foronjy, P. O. Ochieng, M. A. Salathe et al., "Protein tyrosine phosphatase $1 \mathrm{~B}$ negatively regulates S100A9-mediated lung damage during respiratory syncytial virus exacerbations," Mucosal Immunology, vol. 9, no. 5, pp. 1317-1329, 2016.

[53] T. J. Haw, M. R. Starkey, P. M. Nair et al., "A pathogenic role for tumor necrosis factor-related apoptosis-inducing ligand in chronic obstructive pulmonary disease," Mucosal Immunology, vol. 9, no. 4, pp. 859-872, 2016.

[54] P. Geraghty, E. Eden, M. Pillai, M. Campos, N. G. McElvaney, and R. F. Foronjy, " $\alpha 1$-antitrypsin activates protein phosphatase 2A to counter lung inflammatory responses," American Journal of Respiratory and Critical Care Medicine, vol. 190, no. 11, pp. 1229-1242, 2014.

[55] C. Van Hoof and J. Goris, "Phosphatases in apoptosis: to be or not to be, PP2A is in the heart of the question," Biochimica et Biophysica Acta, vol. 1640, no. 2-3, pp. 97-104, 2003.

[56] M. Cano, L. Wang, J. Wan et al., "Oxidative stress induces mitochondrial dysfunction and a protective unfolded protein response in RPE cells," Free Radical Biology and Medicine, vol. 69, pp. 1-14, 2014.

[57] C.-M. Chen, C.-T. Wu, C.-K. Chiang, B.-W. Liao, and S.-H. Liu, "C/EBP homologous protein (CHOP) deficiency aggravates hippocampal cell apoptosis and impairs memory performance," PLoS ONE, vol. 7, no. 7, Article ID e40801, 2012.

[58] V. Esposito, F. Grosjean, J. Tan et al., "CHOP deficiency results in elevated lipopolysaccharide-induced inflammation and kidney injury," American Journal of Physiology - Renal Physiology, vol. 304, no. 4, pp. F440-F450, 2013. 


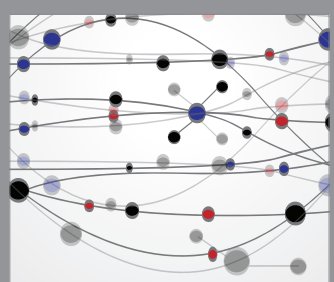

The Scientific World Journal
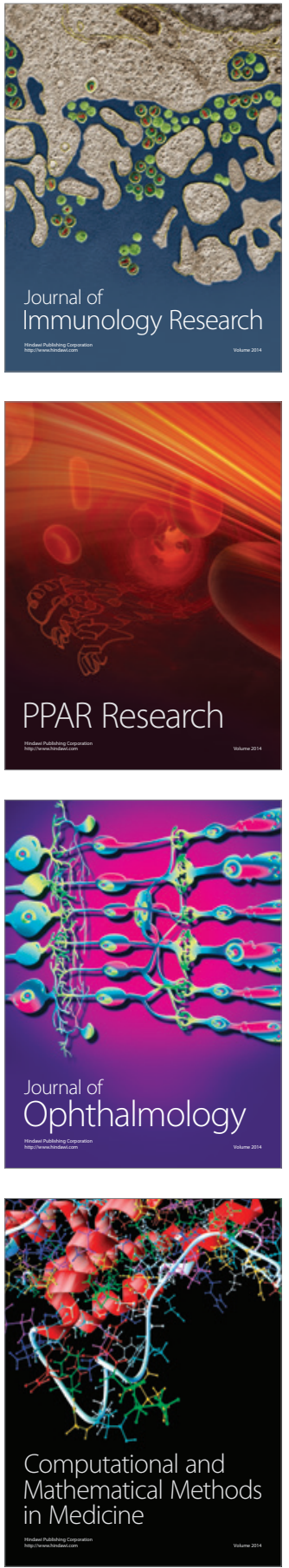

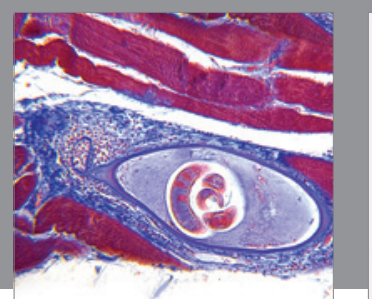

Gastroenterology Research and Practice

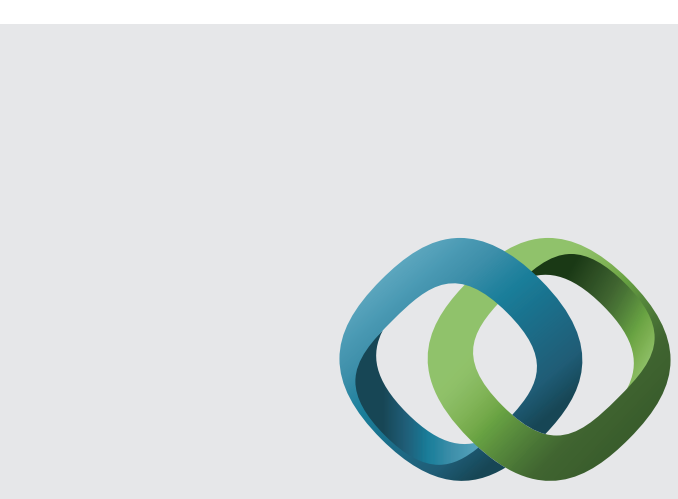

\section{Hindawi}

Submit your manuscripts at

http://www.hindawi.com
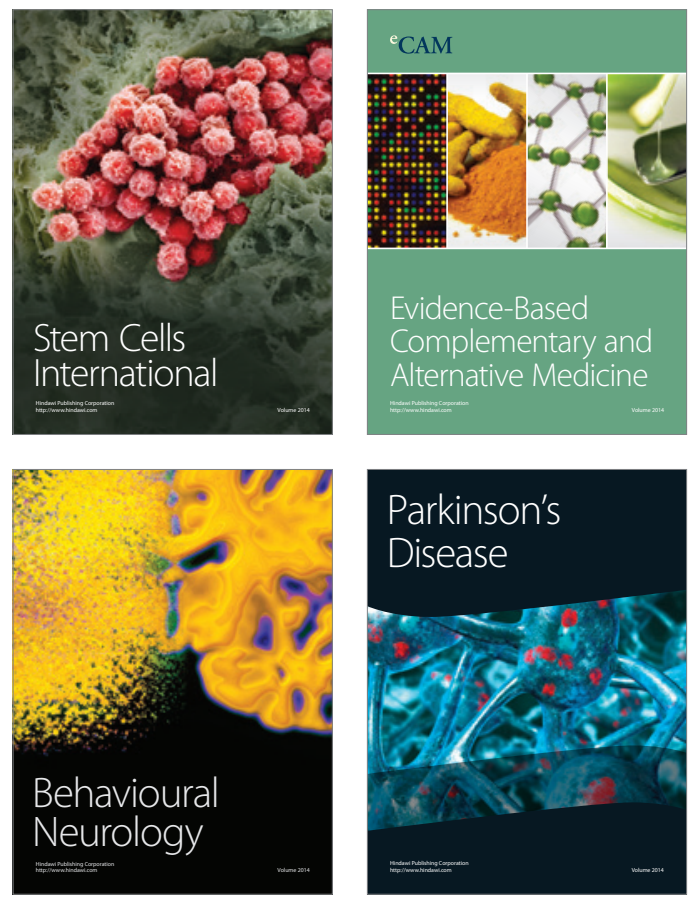
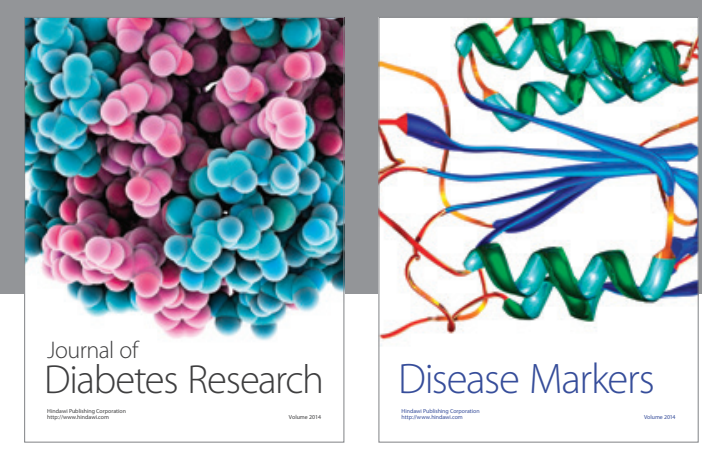

Disease Markers
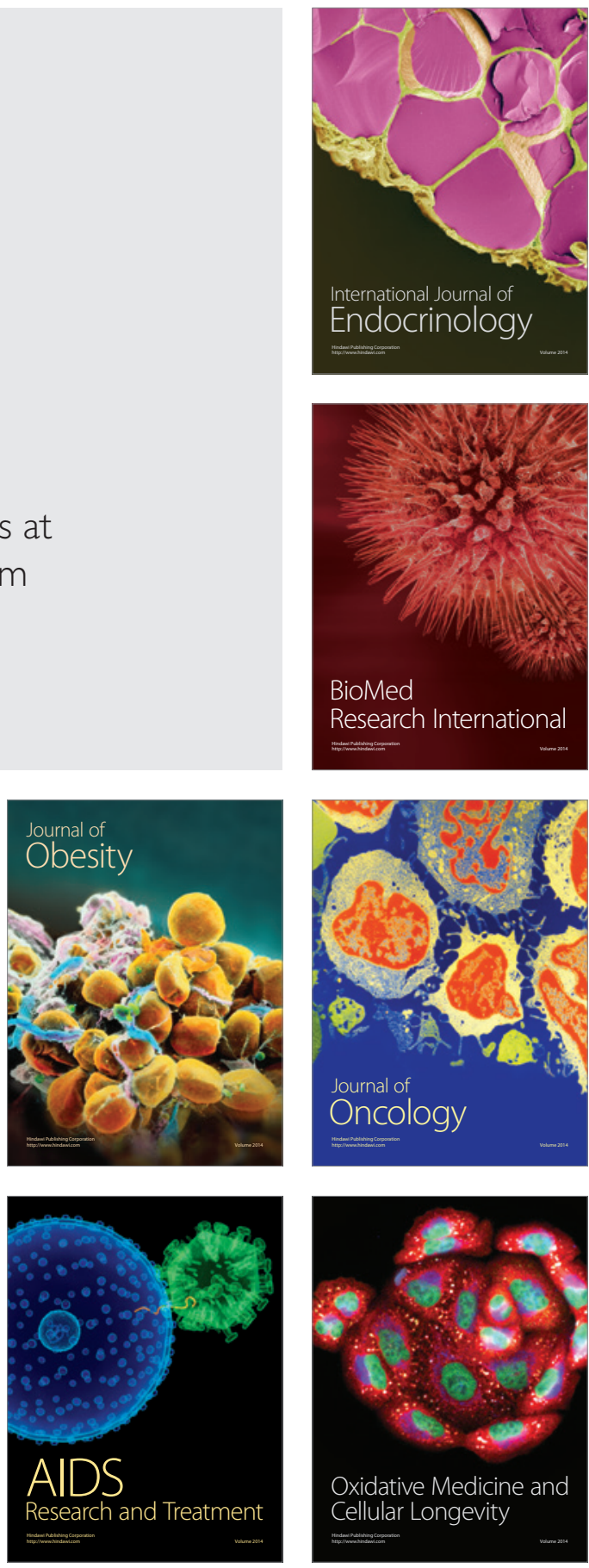